\title{
LOCAL LUMINOUS INFRARED GALAXIES. III. CO-EVOLUTION OF BLACK HOLE GROWTH AND STAR FORMATION ACTIVITY?*
}

\author{
Almudena Alonso-Herrero ${ }^{1,7}$, Miguel Pereira-Santaella ${ }^{2}$, George H. Rieke ${ }^{3}$, \\ Aleksandar M. Diamond-Stanic ${ }^{4}$, Yiping Wang ${ }^{5}$, Antonio Hernán-Caballero ${ }^{1}$, and Dimitra Rigopoulou 6 \\ ${ }^{1}$ Instituto de Física de Cantabria, CSIC-Universidad de Cantabria, E-39005 Santander, Spain \\ 2 Istituto di Astrofisica e Planetologia Spaziali, INAF-IAPS, I-00133 Rome, Italy \\ ${ }^{3}$ Steward Observatory, University of Arizona, Tucson, AZ 85721, USA \\ ${ }^{4}$ Center for Astrophysics and Space Sciences, University of California, San Diego, La Jolla, CA 92093, USA \\ ${ }^{5}$ National Astronomical Observatories, Chaoyang District, Beijing 100012, China \\ ${ }^{6}$ Astrophysics Department, University of Oxford, Oxford OX1 3RH, UK \\ Received 2012 October 31; accepted 2013 January 16; published 2013 February 19
}

\begin{abstract}
Local luminous infrared (IR) galaxies (LIRGs) have both high star formation rates (SFR) and a high AGN (Seyfert and AGN/starburst composite) incidence. Therefore, they are ideal candidates to explore the co-evolution of black hole $(\mathrm{BH})$ growth and star formation (SF) activity, not necessarily associated with major mergers. Here, we use Spitzer/IRS spectroscopy of a complete volume-limited sample of local LIRGs (distances of $<78 \mathrm{Mpc}$ ). We estimate typical BH masses of $3 \times 10^{7} M_{\odot}$ using [Ne III] $15.56 \mu \mathrm{m}$ and optical [O III] $\lambda 5007$ gas velocity dispersions and literature stellar velocity dispersions. We find that in a large fraction of local LIRGs, the current SFR is taking place not only in the inner nuclear $\sim 1.5 \mathrm{kpc}$ region, as estimated from the nuclear $11.3 \mu \mathrm{m}$ PAH luminosities, but also in the host galaxy. We next use the ratios between the SFRs and BH accretion rates (BHAR) to study whether the SF activity and BH growth are contemporaneous in local LIRGs. On average, local LIRGs have SFR to BHAR ratios higher than those of optically selected Seyferts of similar active galactic nucleus (AGN) luminosities. However, the majority of the IR-bright galaxies in the revised-Shapley-Ames Seyfert sample behave like local LIRGs. Moreover, the AGN incidence tends to be higher in local LIRGs with the lowest SFRs. All of this suggests that in local LIRGs there is a distinct IR-bright star-forming phase taking place prior to the bulk of the current BH growth (i.e., AGN phase). The latter is reflected first as a composite and then as a Seyfert, and later as a non-LIRG optically identified Seyfert nucleus with moderate SF in its host galaxy.
\end{abstract}

Key words: galaxies: nuclei - galaxies: Seyfert - infrared: galaxies

Online-only material: color figures

\section{INTRODUCTION}

One of the most fundamental relations in extragalactic astronomy is that, at least in the local universe, the masses of supermassive black holes (BHs, with masses $M_{\mathrm{BH}}>10^{6} M_{\odot}$ ) correlate with the stellar mass and velocity dispersion of the bulges of their host galaxies (see, e.g., Magorrian et al. 1998; Gebhardt et al. 2000; Marconi \& Hunt 2003; Häring \& Rix 2004). This seems to imply that bulges and supermassive BHs evolve together and regulate each other.

Mergers of gas-rich galaxies are efficient both in producing elevated star formation rates (SFR) and transporting gas to the nuclear region to allow for $\mathrm{BH}$ growth. If sufficient matter becomes available very close to the nuclear $\mathrm{BH}$ and is accreted, then the nucleus of the galaxy will shine with enormous power as an active galactic nucleus (AGN; Lynden-Bell 1969). In parallel, and probably before the fully developed AGN phase, the merger will trigger a high rate of star formation (SF) leading to a luminous infrared (IR)-active phase (Sanders et al. 1988), either as a luminous IR galaxy (LIRG) or ultraluminous IR galaxy (ULIRG). These are defined as having an IR 8-1000 $\mu \mathrm{m}$ luminosity $L_{\mathrm{IR}}>10^{11} L_{\odot}$ or $L_{\mathrm{IR}}>10^{12} L_{\odot}$, respectively (see Sanders \& Mirabel 1996, for a review).

\footnotetext{
* This work is based on observations made with the Spitzer Space Telescope, which is operated by the Jet Propulsion Laboratory, California Institute of Technology under NASA contract 1407.

7 Augusto González Linares Senior Research Fellow.
}

As an alternative to this sequence, Kormendy et al. (2011) proposed that BHs in bulgeless galaxies and in galaxies with pseudobulges grow through phases of low-level Seyfert-like activity. Such growth is believed to be driven stochastically by local processes (secular processes), and thus it would not have a global impact on the host galaxy structure (see also Hopkins et al. 2008). Moreover, there is now evidence of two fundamentally different modes of $\mathrm{BH}$ growth at work in earlyand late-type galaxies in the local universe (Schawinski et al. 2010). The role of moderate luminosity AGNs that reside in late-type galaxies and do not involve any recent major mergers is not clear and needs further investigation.

LIRGs are powered by both AGN and SF activity (Sanders \& Mirabel 1996). Alonso-Herrero et al. (2012) showed that while an AGN accompanies SF activity in a large proportion of local LIRGs, in most cases the AGNs are not energetically important. This is because the AGNs hosted in local LIRGs have Seyfertlike luminosities and thus their bolometric contribution to the total IR luminosity is small (typically 5\%). Therefore, the IR luminosities of LIRGs imply SFRs in the range 11-110 $M_{\odot} \mathrm{yr}^{-1}$ using the Kennicutt (1998) prescription converted to a Kroupa (2012) initial mass function (IMF).

It is reasonable to assume that the same gas that is used to form stars in the host galaxy can also be used to feed the AGN provided that there is a mechanism able to transport the gas to the inner region (on scales of less than $0.1 \mathrm{pc}$ ) of the galaxy (see, e.g., the review of Alexander \& Hickox 2012). Indeed, it is 
now apparent that there is a relation between SF activity on subkiloparsec and bulge scales and the $\mathrm{BH}$ accretion rate (BHAR), as shown by observations and numerical simulations (Heckman et al. 2004; Hopkins \& Quataert 2010; Diamond-Stanic \& Rieke 2012). This relation is also predicted to be present, although with a lower significance, for the integrated SFR of the galaxy (Hopkins \& Quataert 2010). Therefore, LIRGs show propitious conditions to study the co-evolution of SF activity and $\mathrm{BH}$ growth as they show both high integrated and nuclear SFRs (Alonso-Herrero et al. 2006) and a high occurrence of AGNs and composite nuclei (60\%-70\%; Yuan et al. 2010; AlonsoHerrero et al. 2012).

In this paper, we study the co-evolution of the SFR activity and the $\mathrm{BH}$ growth in the complete volume-limited sample of local LIRGs of Alonso-Herrero et al. (2006, 2012). We use Spitzer Infrared Spectrograph (IRS; Houck et al. 2004) observations to estimate the nuclear $(\sim 1.5 \mathrm{kpc})$ SFRs and compare them with the integrated values from the IR luminosities. We look for spectroscopically resolved [Ne III] $15.56 \mu \mathrm{m}$ lines. Assuming that this line is produced in the narrow line region (NLR) of the AGN, we measure its velocity dispersion and use the Dasyra et al. (2011) relations to obtain the masses of the BHs hosted in local LIRGs. We complement these with observations of the optical line [O III] $\lambda 5007$ and literature values of the stellar velocity dispersion $\sigma_{*}$ to obtain further estimates of the $\mathrm{BH}$ masses in local LIRGs. All of this information allows us to determine the ratios between the SFR (both nuclear and integrated) and the BHAR in local LIRGs and compare them with those of optically selected Seyfert galaxies. This comparison lets us explore the role of the LIRG phase in the growth of BHs in the local universe. Throughout this paper, we assume the following cosmology: $H_{0}=70 \mathrm{~km} \mathrm{~s}^{-1} \mathrm{Mpc}^{-1}$, $\Omega_{M}=0.3$, and $\Omega_{\Lambda}=0.7$.

\section{SAMPLE, OBSERVATIONS, AND DATA REDUCTION}

\subsection{The Sample of Local LIRGs}

For this work, we use the complete volume-limited sample of local LIRGs that was presented and discussed in detail by Alonso-Herrero et al. (2006, 2012). We drew the sample from the IRAS Revised Bright Galaxy Sample (RBGS; Sanders et al. $2003)$ to include all of the sources with $\log \left(L_{\mathrm{IR}} / L_{\odot}\right) \geqslant 11.05$ and $v_{\text {hel }}=2750-5300 \mathrm{~km} \mathrm{~s}^{-1}$. The sample is composed of 45 IRAS systems, with 8 containing multiple nuclei. Therefore, the sample includes 53 individual galaxies. For the assumed cosmology, the distances are in the range $\simeq 40-78 \mathrm{Mpc}$, with a median value of $65 \mathrm{Mpc}$. The IR luminosities of the individual galaxies $^{8}$ are in the range $\log \left(L_{\mathrm{IR}} / L_{\odot}\right)=10.64-11.67$, with a median value of $\log \left(L_{\mathrm{IR}} / L_{\odot}\right)=11.12$. All of the relevant information for the sample can be found in Table 1 of AlonsoHerrero et al. (2012).

Combining a number of optical and mid-infrared (midIR) indicators, Alonso-Herrero et al. (2012) derived an AGN detection rate of $\sim 62 \%$ for this complete volume-limited sample of local LIRGs. The derived AGN bolometric luminosities, from the mid-IR spectral decomposition and/or X-ray observations, are in the range $L_{\text {bol }}(\mathrm{AGN})=(0.4-50) \times 10^{43} \mathrm{erg} \mathrm{s}^{-1}$, with a median of $\sim 1.4 \times 10^{43} \mathrm{erg} \mathrm{s}^{-1}$ (Pereira-Santaella et al. 2011; Alonso-Herrero et al. 2012). However, these AGN are overall only responsible for $\sim 5 \%$ of the IR luminosity emitted by local

\footnotetext{
8 Galaxies significantly below the selection luminosity are members of multiple systems with total luminosities that satisfy the selection criterion.
}

Table 1

Spitzer/IRS Calibration Sources

\begin{tabular}{lc}
\hline \hline Name & AOR \\
\hline PNG043.1+37.7 & 4109056 \\
PNG011.7-00.6 & 4109568 \\
PNG025.8-17.9 & 4110080 \\
PNG265.7+04.1 & 4111104 \\
PNG206.4-40.5 & 4111616 \\
PNG285.7-14.9 & 4112128 \\
PNG054.1-12.1 & 4112640 \\
PNG009.4-05.0 & 4114176 \\
PNG342.1+10.8 & 4115200 \\
PNG316.1+08.4 & 4116224 \\
Calwav-22B-NGC 7027 & 15343104 \\
IRSS-SEPN-N7293-0001 & 15752960
\end{tabular}

LIRGs, although the AGN contribution varies from source to source (see Imanishi et al. 2010; Alonso-Herrero et al. 2012). This is in good agreement with Petric et al. (2011) for The Great Observatories All-Sky LIRG Survey (GOALS; Armus et al. 2009).

\subsection{Spitzer/IRS Observations}

\subsubsection{Observations}

We retrieved Spitzer/IRS (Houck et al. 2004) spectroscopy of the sample of LIRGs taken with the high-resolution $(R \sim 600)$ short-high $(\mathrm{SH})$ and long-high $(\mathrm{LH})$ modules that cover the 9.9-19.6 $\mu \mathrm{m}$ and $18.7-37.2 \mu \mathrm{m}$ spectral ranges, respectively. Details on the program IDs, the observations, and data reduction of the galaxies, including those from GOALS (Armus et al. 2009), are given in Alonso-Herrero et al. (2012) and PereiraSantaella et al. (2010a, 2010b). We note that for various reasons, two galaxies in this volume-limited sample were not observed with the Spitzer/IRS high spectral resolution modules. We extracted the nuclear spectra assuming a point-source calibration.

We also retrieved SH and LH spectroscopy from the Spitzer archive of 12 calibration sources and planetary nebulae to obtain an accurate estimate of the spectral resolution using finestructure lines (see Section 2.2.3). The names and corresponding astronomical observation request (AOR) numbers are give in Table 1. We reduced the data as for the sample of local LIRGs.

\subsubsection{Line Fitting}

For the fine-structure lines, we used Gaussians to measure the line flux, equivalent width (EW), and full width at halfmaximum (FWHM) and a first-order polynomial to fit the local continuum (see Pereira-Santaella et al. 2010a, for more details). The fluxes of the [Ne II] $12.81 \mu \mathrm{m}$, [Ne III] $15.56 \mu \mathrm{m}$, and [O IV] $25.89 \mu \mathrm{m}$ lines can be found in Pereira-Santaella et al. (2010b) and Alonso-Herrero et al. (2012). For the $11.3 \mu \mathrm{m}$ polycyclic aromatic hydrocarbon (PAH) feature, we first fitted the local continuum using a linear fit between 10.6 and $11.8 \mu \mathrm{m}$. After subtracting the continuum, we integrated the flux in the wavelength range between 10.8 and $11.6 \mu \mathrm{m}$. We list the fluxes, $\mathrm{EW}$, and corresponding errors of the $11.3 \mu \mathrm{m}$ PAH feature in Table 2.

\subsubsection{Spectrally Resolved [Ne III] $15.56 \mu \mathrm{m}$ Lines}

The velocity dispersion of the ionized gas in the NLR allows measuring the $\mathrm{BH}$ mass $\left(M_{\mathrm{BH}}\right)$ in an AGN supplementing 
Table 2

Fluxes and EW of the Nuclear $11.3 \mu \mathrm{m}$ PAH Feature

\begin{tabular}{|c|c|c|}
\hline Galaxy & Flux & EW \\
\hline NGC 23 & $430.4 \pm 8.5$ & $0.78 \pm 0.05$ \\
\hline MCG+12-02-001 & $396.8 \pm 7.9$ & $0.42 \pm 0.01$ \\
\hline NGC 633 & $166.1 \pm 4.0$ & $0.61 \pm 0.04$ \\
\hline ESO 297-G012 & $128.4 \pm 3.5$ & $0.60 \pm 0.05$ \\
\hline UGC 01845 & $347.5 \pm 5.2$ & $0.74 \pm 0.03$ \\
\hline UGC 02982 & $379 \pm 35$ & $0.84 \pm 0.15$ \\
\hline CGCG 468-002 NED01 & $59.6 \pm 5.6$ & $0.24 \pm 0.03$ \\
\hline CGCG 468-002 NED02 & $78.4 \pm 7.7$ & $0.46 \pm 0.07$ \\
\hline UGC 03351 & $275.5 \pm 8.3$ & $0.78 \pm 0.06$ \\
\hline NGC 2369 & $357.1 \pm 3.9$ & $0.58 \pm 0.02$ \\
\hline NGC 2388 & $428 \pm 14$ & $0.53 \pm 0.04$ \\
\hline MCG+02-20-003 & $149.5 \pm 4.1$ & $0.70 \pm 0.06$ \\
\hline NGC 3110 & $299.0 \pm 4.0$ & $0.80 \pm 0.03$ \\
\hline NGC 3256 & $1547.2 \pm 4.8$ & $0.556 \pm 0.004$ \\
\hline ESO 264-G057 & $165 \pm 13$ & $0.76 \pm 0.11$ \\
\hline IC 694 & $446 \pm 16$ & $0.38 \pm 0.02$ \\
\hline NGC 3690 & $308 \pm 25$ & $0.09 \pm 0.01$ \\
\hline ESO 320-G030 & $450.3 \pm 4.6$ & $0.78 \pm 0.03$ \\
\hline MCG-02-33-098 W & $99.0 \pm 4.2$ & $0.27 \pm 0.02$ \\
\hline MCG-02-33-098 E & $102.3 \pm 6.8$ & $0.62 \pm 0.12$ \\
\hline IC 860 & $43.2 \pm 2.2$ & $0.44 \pm 0.04$ \\
\hline MCG-03-34-064 & $23.0 \pm 3.2$ & $0.013 \pm 0.002$ \\
\hline NGC 5135 & $376.9 \pm 4.0$ & $0.46 \pm 0.01$ \\
\hline ESO 173-G015 & $434 \pm 31$ & $0.53 \pm 0.06$ \\
\hline IC 4280 & $249.4 \pm 7.0$ & $0.80 \pm 0.04$ \\
\hline UGC 08739 & $146 \pm 41$ & $0.84 \pm 0.38$ \\
\hline ESO 221-IG010 & $259.9 \pm 9.4$ & $0.63 \pm 0.05$ \\
\hline NGC 5653 & $385 \pm 10$ & $0.81 \pm 0.07$ \\
\hline NGC 5734 & $402 \pm 11$ & $0.80 \pm 0.06$ \\
\hline NGC 5743 & $251 \pm 14$ & $0.75 \pm 0.12$ \\
\hline IC $4518 \mathrm{E}$ & $64.6 \pm 1.4$ & $0.62 \pm 0.05$ \\
\hline IC $4518 \mathrm{~W}$ & $62.6 \pm 5.9$ & $0.16 \pm 0.02$ \\
\hline Zw 049.057 & $62.3 \pm 4.5$ & $0.96 \pm 0.23$ \\
\hline NGC 5936 & $240.0 \pm 6.6$ & $0.67 \pm 0.05$ \\
\hline NGC 5990 & $243 \pm 56$ & $0.29 \pm 0.09$ \\
\hline NGC 6156 & $141 \pm 16$ & $0.22 \pm 0.03$ \\
\hline IRAS 17138-1017 & $307.6 \pm 4.3$ & $0.53 \pm 0.02$ \\
\hline IRAS $17578-0400$ & $177 \pm 10$ & $0.76 \pm 0.09$ \\
\hline IC 4687 & $478.2 \pm 4.2$ & $0.70 \pm 0.02$ \\
\hline IC 4734 & $195.1 \pm 3.8$ & $0.59 \pm 0.03$ \\
\hline NGC 6701 & $242.2 \pm 4.2$ & $0.67 \pm 0.03$ \\
\hline MCG +04-48-002 & $394 \pm 33$ & $0.73 \pm 0.11$ \\
\hline NGC 7130 & $230.5 \pm 3.7$ & $0.36 \pm 0.01$ \\
\hline IC 5179 & $391.9 \pm 3.6$ & $0.68 \pm 0.02$ \\
\hline NGC 7469 & $583.5 \pm 7.7$ & $0.237 \pm 0.005$ \\
\hline NGC 7591 & $142 \pm 12$ & $0.36 \pm 0.06$ \\
\hline NGC 7679 & $363 \pm 16$ & $0.61 \pm 0.05$ \\
\hline NGC 7769 & $85 \pm 13$ & $0.57 \pm 0.21$ \\
\hline NGC 7770 & $131 \pm 18$ & $0.47 \pm 0.13$ \\
\hline NGC 7771 & $359 \pm 16$ & $0.65 \pm 0.07$ \\
\hline
\end{tabular}

Notes. The fluxes are in units of $10^{-14} \mathrm{erg} \mathrm{cm}^{-2} \mathrm{~s}^{-1}$ and the EW are in units of $\mu \mathrm{m}$.

reverberation mapping techniques. As discussed by Greene \& Ho (2005), the NLR is sufficiently compact to be illuminated by the AGN, while large enough to feel the gravitational potential of the bulge. Therefore, there is a relatively good correlation between the gas and the stellar velocity dispersion around local AGN. In the mid-IR, Dasyra et al. $(2008,2011)$ showed that the velocity dispersion of the fine-structure lines [S IV] $10.51 \mu \mathrm{m}$, [Ne III] $15.56 \mu \mathrm{m}$, [Ne v] $14.32 \mu \mathrm{m}$, and [O IV] $25.89 \mu \mathrm{m}$ of AGN are well correlated with $M_{\mathrm{BH}}$.
For our Spitzer/IRS spectra, we focus on the [Ne III] $15.56 \mu \mathrm{m}$ line to look for spectrally resolved line profiles. In AGNs, the flux and luminosity of this fine-structure line are found to correlate well with those of the mid-IR [Ne v] lines at 14.3 and $24.3 \mu \mathrm{m}$ (Gorjian et al. 2007; Pereira-Santaella et al. $2010 \mathrm{~b})$. The high-ionization potential of the [Ne v] lines indicates that they are mostly excited by AGNs and thus, the [Ne III] $15.56 \mu \mathrm{m}$ emission is likely to be as well. When compared with other mid-IR fine-structure lines with relatively high ionization potentials (e.g., [S IV] $10.51 \mu \mathrm{m}$, [O IV] $25.89 \mu \mathrm{m}$, and the [Ne V] lines), the [Ne III] $15.56 \mu \mathrm{m}$ line is a good compromise between ionization potential, critical density, ${ }^{9}$ intensity of the line, and a clean region of the mid-IR spectrum for accurate measurements.

Using the calibration sources (Section 2.2.1), we obtained 12 independent measurements of the unresolved width of the [Ne III] $15.56 \mu \mathrm{m}$ line and inferred an instrumental width of $\mathrm{FWHM}_{\text {inst }}=0.02618 \pm 0.0007 \mu \mathrm{m}$. This is equivalent to a spectral resolution of $R=\lambda / \mathrm{FWHM}_{\text {inst }} \sim 595 \pm 16$ and an instrumental velocity dispersion of $\sigma_{\text {inst }}=215 \pm 6 \mathrm{~km} \mathrm{~s}^{-1}$ at the wavelength of this line. While the derived spectral resolution is very similar to that from Dasyra et al. (2011), the standard deviation of our measurements is much lower. Dasyra et al. (2011) obtained $\sigma_{\text {inst }}$ as the average of the values of the highexcitation fine-structure lines of a sample of AGNs that were deemed not to be resolved by Spitzer/IRS with their method. By doing so, they probably also included measurements of barely resolved lines. This would therefore explain the higher dispersion of their instrumental value.

For the LIRGs, we need a criterion to assess whether the lines are resolved or not since a significant number of sources have measured widths slightly larger than the instrumental resolution. Figure 1 shows the distribution of the measured FWHM of the [Ne III] $15.56 \mu \mathrm{m}$ line for the sample of LIRGs compared with those of the calibration sources. To avoid analyzing barely resolved lines, we adopt the following criterion for the velocity dispersion to determine whether a line is clearly resolved: $\left(\sigma_{\text {obs }}-\sigma_{\text {inst }}\right)>3 \times \sqrt{\epsilon_{\mathrm{m}}^{2}+\epsilon_{\text {inst }}^{2}}$, where $\sigma_{\text {inst }}$ and $\epsilon_{\text {inst }}$ are the instrumental resolution and its standard deviation, and $\sigma_{\text {obs }}$ and $\epsilon_{\mathrm{m}}$ are the measured values for the LIRGs. The total error of the observations $\epsilon_{\mathrm{obs}}$ includes both the error in the measurement $\epsilon_{\mathrm{m}}$ and that associated with the instrumental value, that is, $\epsilon_{\mathrm{obs}}=\left(\epsilon_{\mathrm{m}}^{2}+\epsilon_{\mathrm{inst}}^{2}\right)^{1 / 2}$.

Using the criterion described above, we found that 19 LIRGs in our sample show resolved [Ne III] $15.56 \mu \mathrm{m}$ lines. Most are LIRGs classified as Seyfert (Figure 1) and/or are [Ne v] emitters. Those nuclei classified as HII, on the other hand, tend to show FWHM close to the instrumental resolution. Composite nuclei have FWHM between those of unresolved lines and the clearly resolved [Ne III] $15.56 \mu \mathrm{m}$ lines. For those LIRGs deemed to have unresolved [Ne III] $15.56 \mu \mathrm{m}$ lines, the average measured FWMH of the [Ne III] $15.56 \mu \mathrm{m}$ line is $0.02776 \pm 0.0017 \mu \mathrm{m}$. That is, the observed values for these lines are on average $2 \sigma$ above the instrumental resolution.

Table 3 lists the intrinsic values of the velocity dispersion for the LIRGs with resolved [Ne III] $15.56 \mu \mathrm{m}$ lines as well as their spectroscopic classes from optical spectroscopy and/or mid-IR indicators (Alonso-Herrero et al. 2012). The velocity dispersions (corrected for instrumental resolution) are between 102 and $368 \mathrm{~km} \mathrm{~s}^{-1}$. For galaxies in our sample with literature values

\footnotetext{
9 For reference, the [Ne III] $15.56 \mu \mathrm{m}$ line has a slightly higher ionization potential than the optical [O III] $\lambda 5007$ emission line but the optical line has a slightly higher critical density (see Figure 6 of Dasyra et al. 2011).
} 


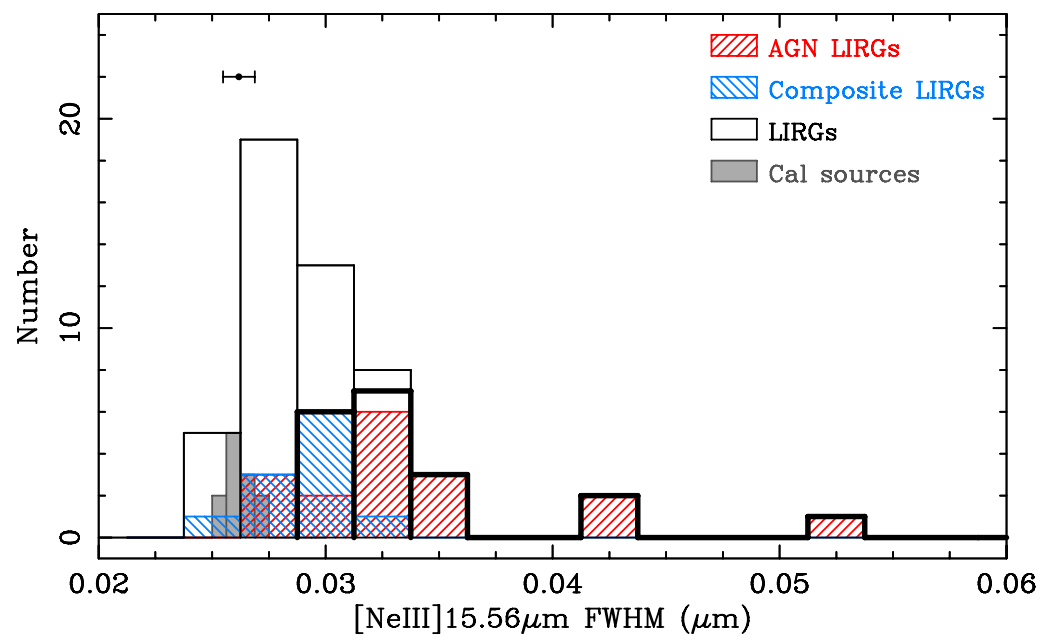

Figure 1. Distribution of the observed (not corrected for instrumental resolution) FWHMs of the [Ne III] $15.56 \mu \mathrm{m}$ line of local LIRGs compared with those of the calibration sources. The latter are assumed to provide the instrumental resolution at the wavelength of the line. The dot represents the average value of the FWHM of the calibration sources with the corresponding $1 \sigma$ error. We marked those LIRG nuclei classified as AGNs (Seyfert and/or [Ne v] emitters) and composites. The rest are H II-like or have no classification. The thick line histogram represents the observed FWHM of those galaxies deemed to have spectroscopically resolved lines.

(A color version of this figure is available in the online journal.)

Table 3

Velocity Dispersions and BH Masses

\begin{tabular}{|c|c|c|c|c|c|c|c|}
\hline Galaxy & Class & $\begin{array}{c}\sigma([\mathrm{Ne} \mathrm{III]}]) \\
\left(\mathrm{km} \mathrm{s}^{-1}\right)\end{array}$ & $\begin{array}{c}\sigma([\mathrm{O} \mathrm{III}])_{\mathrm{n}} \\
\left(\mathrm{km} \mathrm{s}^{-1}\right)\end{array}$ & $\begin{array}{c}\sigma([\mathrm{O} \mathrm{III]}])_{\mathrm{b}} \\
\left(\mathrm{km} \mathrm{s}^{-1}\right)\end{array}$ & $\begin{array}{c}\sigma_{*} \\
\left(\mathrm{~km} \mathrm{~s}^{-1}\right)\end{array}$ & Reference & $\begin{array}{c}\log M_{\mathrm{BH}} \\
\left(M_{\odot}\right)\end{array}$ \\
\hline \multicolumn{8}{|c|}{ Seyfert nuclei and $[\mathrm{Ne} \mathrm{v}]$ emitters } \\
\hline CGCG 468-002-NED01 & Sy2 & $135 \pm 12$ & $218 \pm 22$ & $\ldots$ & $\ldots$ & $\ldots$ & 8.06 \\
\hline NGC 3690 & Sy2 & $167 \pm 10$ & $\ldots$ & $\ldots$ & $144 \pm 11$ & 1 & 7.52 \\
\hline MCG -03-34-064 & Sy1 & $368 \pm 18$ & $249 \pm 10$ & $656 \pm 26$ & 155 & 2 & 7.65 \\
\hline NGC 5135 & Sy2 & $138 \pm 9$ & $127 \pm 9$ & $305 \pm 21$ & $124 \pm 6$ & 3 & 7.24 \\
\hline IC 4518W & Sy2 & $156 \pm 12$ & $\ldots$ & $\ldots$ & $\ldots$ & $\ldots$ & 7.48 \\
\hline NGC 5990 & Sy2 & $264 \pm 11$ & $\ldots$ & $\ldots$ & $\ldots$ & $\ldots$ & $<8.45$ \\
\hline NGC 6156 & {$[\mathrm{Ne} \mathrm{v}]$} & $160 \pm 13$ & $\ldots$ & $\ldots$ & $\ldots$ & $\ldots$ & 7.52 \\
\hline MCG+04-48-002 & {$[\mathrm{Ne} \mathrm{v}]$} & $158 \pm 7$ & $\ldots$ & $\ldots$ & $\ldots$ & $\ldots$ & 7.50 \\
\hline NGC 7130 & Sy2: & $259 \pm 16$ & $130 \pm 9$ & $463 \pm 32$ & $147 \pm 5$ & 3 & 7.55 \\
\hline NGC 7469 & Sy1 & $182 \pm 13$ & $145 \pm 7$ & $336 \pm 17$ & $152 \pm 16$ & 4 & 7.61 \\
\hline NGC 7591 & Sy2: & $169 \pm 17$ & $\ldots$ & $\ldots$ & $\ldots$ & $\ldots$ & 7.62 \\
\hline NGC 7679 & Sy1/Sy2 & $102 \pm 7$ & $176^{\mathrm{a}}$ & $\ldots$ & 96 & 2 & 6.77 \\
\hline \multicolumn{8}{|c|}{ Composite and $\mathrm{H}$ II nuclei } \\
\hline NGC 1614 & Composite & $157 \pm 10$ & $\ldots$ & $\ldots$ & $164 \pm 8$ & 5 & 7.75 \\
\hline NGC 2369 & Composite & $133 \pm 8$ & $146 \pm 15$ & $\ldots$ & $\ldots$ & $\ldots$ & $\ldots$ \\
\hline IC 694 & LINER & $<70$ & $\ldots$ & $\ldots$ & $141 \pm 17$ & 5 & 7.48 \\
\hline NGC 3256 & H II & $<112$ & $\ldots$ & $\ldots$ & $100 \pm 6$ & 3 & 6.84 \\
\hline MCG-02-33-098W & Composite & $103 \pm 9$ & $\cdots$ & $\ldots$ & $\ldots$ & $\ldots$ & $\ldots$ \\
\hline ESO 173-G015 & $\ldots$ & $102 \pm 7$ & $\ldots$ & $\ldots$ & $\ldots$ & $\ldots$ & $\cdots$ \\
\hline IC 4280 & H II & $128 \pm 8$ & $\ldots$ & $\ldots$ & $\ldots$ & $\ldots$ & $\ldots$ \\
\hline IRAS $17138-1017 \mathrm{~N}$ & Composite & $<80$ & $\ldots$ & $\ldots$ & $72 \pm 5$ & 6 & 6.24 \\
\hline IRAS $17578-0400$ & $\ldots$ & $119 \pm 8$ & $\ldots$ & $\ldots$ & $\ldots$ & $\ldots$ & $\ldots$ \\
\hline NGC 6701 & Composite & $<159$ & $\cdots$ & $\ldots$ & $150 \pm 20$ & 7 & 7.59 \\
\hline NGC 7771 & Composite & $178 \pm 17$ & $\ldots$ & $\ldots$ & $\ldots$ & $\ldots$ & $\ldots$ \\
\hline
\end{tabular}

Notes. The references for the spectral class of the nuclei are listed in Alonso-Herrero et al. (2012) except for CGCG 468-002-NED01, which is from the newly analyzed optical spectrum (see Section 2.3).

${ }^{a}$ The [O III] velocity dispersion for NGC 7679 is also from Gu et al. (2006).

References. References for $\sigma_{*}$ : (1) Ho et al. 2009; (2) Gu et al. 2006; (3) Garcia-Rissmann et al. 2005; (4) Onken et al. 2004; (5) Hinz \& Rieke 2006; (6) Shier \& Fisher 1998; (7) Márquez et al. 1996.

of the stellar velocity dispersions whose [Ne III] $15.56 \mu \mathrm{m}$ lines are deemed to be unresolved, we provide in this table upper limits to the gas velocity dispersion. In Figure 2, we show the normalized profiles of the $12 \mathrm{Sy} /[\mathrm{Ne}$ v] emitter LIRGs with spectrally resolved [Ne III] $15.56 \mu \mathrm{m}$ lines compared with those of an unresolved profile represented as a Gaussian function.

\subsection{Optical [O III] $\lambda 5007$ Measurements}

We obtained archival optical spectra of six LIRGs classified as AGN or composite from the six-degree Field (6dF) Galaxy Survey (6dfGS; Jones et al. 2004, 2009) and from observations with the FAST spectrograph (Tokarz \& Roll 1997; 

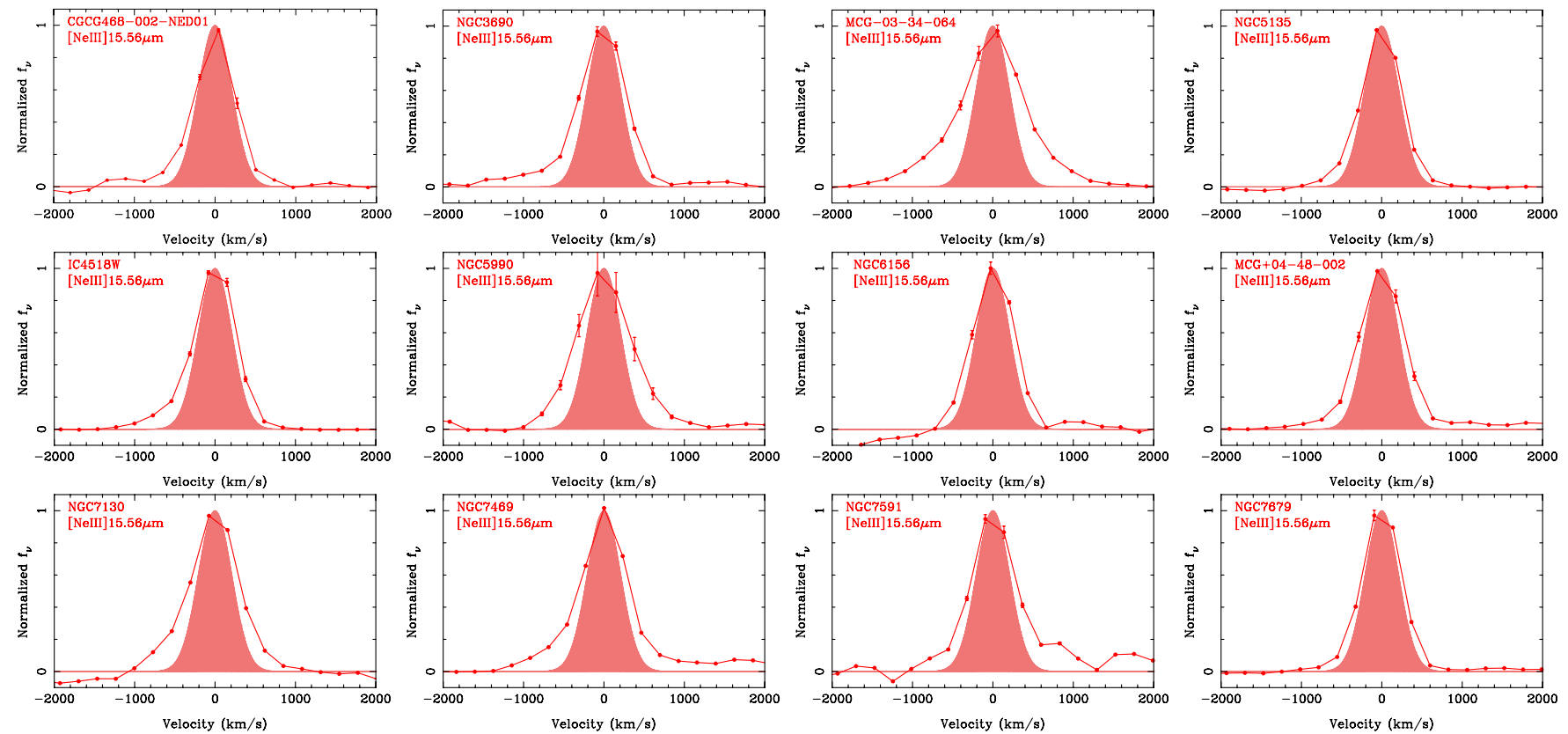

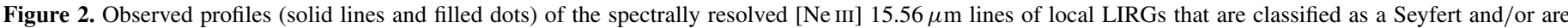

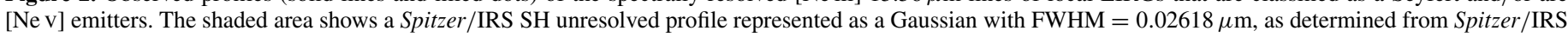
calibration sources (Section 2.2.1).

(A color version of this figure is available in the online journal.)

Fabricant et al. 1998) from the Center for Astrophysics (CfA) telescope data center.

NGC 2369, MCG -03-34-06, NGC 5135, and NGC 7130 were observed with the $6 \mathrm{dF}$ multi-object fiber (angular diameter of 6.7) spectrograph on the United Kingdom Schmidt Telescope (UKST). At the typical distance of these LIRGs, the spectra cover the central $\sim 2 \mathrm{kpc}$. The instrument spectral resolution around the [O III] emission lines is $\sigma_{\text {inst }} \sim 125 \mathrm{~km} \mathrm{~s}^{-1}$. Longslit spectroscopy was available for CGCG 468-002-NED01 and NGC 7469, taken with FAST on the Mt. Hopkins Tillinghast 60 inch telescope as part of different observing programs. The slit width was $3^{\prime \prime}$ and the spectral resolution was $\sigma_{\text {inst }} \sim 75 \mathrm{~km} \mathrm{~s}^{-1}$. None of the spectra are flux calibrated, but they can be used to measure the velocity dispersion of the emission lines as well as their ratios.

We first measured the optical line ratios of CGCG 468002-NED01 since we did not have an optical classification for this galaxy. We classify this galaxy as a Seyfert 2. In PereiraSantaella et al. (2010b) and Alonso-Herrero et al. (2012), we detected the mid-IR [Ne V] lines in this galaxy and measured a high [O IV] $25.89 \mu \mathrm{m} /[\mathrm{Ne}$ II] $12.81 \mu \mathrm{m}$ line ratio. We used both as evidence of the presence of an AGN in this nucleus. The optical classification confirms this.

For all of the galaxies, we modeled the [O III] emission lines at $5007 \AA$ and $4959 \AA$ by fitting a Gaussian profile to each line. The relative position of the Gaussians was fixed according to the rest-frame wavelengths of the two lines. Likewise, the relative intensity was also fixed to the value determined by the atomic parameters. We found that the fit with one component was only satisfactory for CGCG 468-002-NED01 and NGC 2369. For the rest, we had to add a broad component to the [O III] lines. The wavelengths and intensities of the narrow (also called core) component and the broad component were considered independent. To estimate the errors of the velocity dispersions, we used the rms of the continuum adjacent to the line for the $6 \mathrm{dF}$ spectra and the errors of the spectra for the FAST data. The typical errors of the velocity dispersions of the [O III] lines are less than $10 \%$, and are listed for each galaxy in Table 3.

The top panel of Figure 3 shows the galaxies for which the [O III] $\lambda 5007$ line fits required two components, whereas the bottom panel shows the profiles of NGC 2369 and CGCG 468-002-NED01. Table 3 lists the velocity dispersion of the core of the line and the broad component, if present, corrected for instrumental resolution. The velocity dispersions of the core components measured in the four galaxies with double components are in good agreement with literature $\sigma_{*}$ values, except for MCG -03-34-064.

For the four LIRGs with double components, the broad component is blueshifted typically by $50-300 \mathrm{~km} \mathrm{~s}^{-1}$. The presence of such asymmetric or broad blue wings in the [O III] $\lambda 5007$ lines is common in AGNs (see, e.g., Greene \& Ho 2005). The total [O III] emission is dominated by the broad component in MCG-03-34-064 and in NGC 7130, whereas in NGC 5135 and NGC 7469 the core component dominates the emission. In NGC 5135, Bedregal et al. (2009), based on both the relatively broad [Si VI] $1.96 \mu \mathrm{m}$ coronal line and its spatial extent, suggested the existence of different kinematical components and the possible presence of AGN-induced outflows. González Delgado et al. (1998) found blueshifted components in the Ly $\alpha$ and [O III] $\lambda 5007$ lines of both NGC 7130 and NGC 5135. The velocity dispersion of the broad component of the [O III] $\lambda 5007$ line of NGC 7130 is similar to the $\mathrm{H} \alpha$ broad component ( $\sigma \sim 400 \mathrm{~km} \mathrm{~s}^{-1}$; Bellocchi et al. 2012). In NGC 7469, Wilson et al. (1986) also detected two components in the $[\mathrm{O}$ III] $\lambda 5007$ line.

\section{RESULTS}

\subsection{Black Hole Masses}

In Table 3, we list, in addition to the gas velocity dispersions from the [Ne III] $15.56 \mu \mathrm{m}$ and optical [O III] $\lambda 5007$ lines, the stellar velocity dispersion $\sigma_{*}$ values from the literature, when 

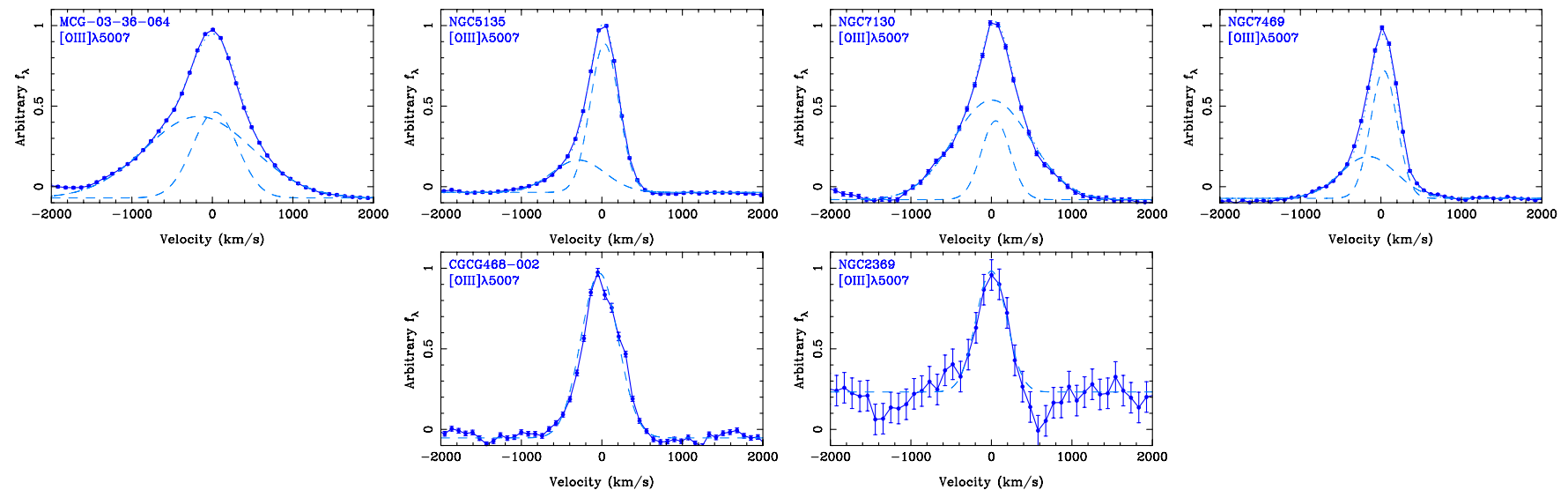

Figure 3. Top panel: observed profiles (solid lines and filled dots) of the [O III] $\lambda 5007$ line fitted with two Gaussian components (dashed lines) as explained in Section 2.3 . Bottom panel: same as top panel but for galaxies whose profiles are fitted with one Gaussian. The plotted errors for the 6dF data (NGC 2369, MGC -03-34-064, NGC 5135, and NGC 7130) are those computed from the rms of the continuum adjacent to the line.

(A color version of this figure is available in the online journal.)

available. We also included in this table $\sigma_{*}$ values from the literature for other LIRGs in our sample (IC 694, NGC 3256, IRAS 17138-1017, and NGC 6701) whose [Ne III] $15.56 \mu \mathrm{m}$ lines appear unresolved at the $\mathrm{SH}$ spectral resolution. We note, however, that in the case of ongoing major mergers, the stellar velocity dispersion versus $\mathrm{BH}$ mass relation might not be applicable.

Dasyra et al. $(2008,2011)$ showed that mid-IR resolved finestructure lines can be used to study the NLR of AGNs. In particular, they demonstrated that the mass of the BH correlates with both the velocity dispersion and the luminosity of the NLR region for a sample of optically selected AGNs. The relation is

$$
\log \left(M_{\mathrm{BH}} / M_{\odot}\right)=-1.82+4.24 \log \left(\sigma_{\mathrm{Ne} \text { III }} / \mathrm{km} \mathrm{s}^{-1}\right) .
$$

Dasyra et al. (2011) fitted a similar relation for the optical [O III] $\lambda 5007$ line:

$$
\log \left(M_{\mathrm{BH}} / M_{\odot}\right)=-1.78+4.24 \log \left(\sigma_{\mathrm{O}} \mathrm{III} / \mathrm{km} \mathrm{s}^{-1}\right) .
$$

For both relations, Dasyra et al. (2011) fixed the slope to the value derived from the correlation between the stellar velocity dispersion and the BH mass by Gültekin et al. (2009):

$$
\log \left(M_{\mathrm{BH}} / M_{\odot}\right)=8.12+4.24 \log \left(\sigma_{*} / 200 \mathrm{~km} \mathrm{~s}^{-1}\right) .
$$

The typical uncertainties on the derived $M_{\mathrm{BH}}$ can be as high as 0.8 dex if using the gas $\sigma$ based on the rms of the relations (Dasyra et al. 2011), and $0.4 \mathrm{dex}$ if using $\sigma_{*}$ (Gültekin et al. 2009).

Before we compute the $\mathrm{BH}$ masses, we compare the gas velocity dispersions for the LIRGs with both [Ne III] $15.56 \mu \mathrm{m}$ and [O III] $\lambda 5007$ measurements. As we saw in Section 2.3, in some cases, the optical line is fitted with two components. For AGN, there is generally a good agreement between $\sigma_{*}$ and the gas velocity dispersion from the narrow component of [O III] (Onken et al. 2004; Greene \& Ho 2005). Therefore, the core of the [O III] $\lambda 5007$ line probes the gravitational movements in the NLR and thus can be used to estimate $M_{\mathrm{BH}}$. On the other hand, the broad components tend to be blueshifted, may be more influenced by the AGN, and are likely produced by outflows. As can be seen from Table 3, the velocity dispersions from the [Ne III] $15.56 \mu \mathrm{m}$ line appear to be intermediate between those of the two optical components. Spectrally resolved blueshifted [Ne III] $15.56 \mu \mathrm{m}$ lines (as well as the [Ne v] lines) have also been reported for local ULIRGs (Spoon \& Holt 2009), and have been interpreted as the result of outflows. Thus, it is possible that in some cases, we might overestimate the $\mathrm{BH}$ masses when using the [Ne III] $15.56 \mu \mathrm{m}$ line if there are several components. The most suspect galaxy in our sample would be NGC 5990, for which $\sigma_{\mathrm{Ne} \text { III }}$ predicts the most massive $\mathrm{BH}$ by far in our sample. We note, however, that the calibration of Dasyra et al. (2011) should take these effects into consideration.

In Table 3, we list $M_{\mathrm{BH}}$ as calculated from, in order of preference, the stellar velocity dispersion, the velocity dispersion of the core of the [O III] line, and the [Ne III] $15.56 \mu \mathrm{m}$ line velocity dispersion. For those LIRGs with no definitive evidence of AGN activity (i.e., classified as composites, H II or unknown), we only list $M_{\mathrm{BH}}$ in the second part of the table if we have a value of $\sigma_{*}$. This is because we cannot be sure whether the [Ne III] $15.56 \mu \mathrm{m}$ emission comes mostly from the NLR or whether it is produced by SF (Pereira-Santaella et al. 2010b).

For those LIRGs hosting an actively accreting $\mathrm{BH}$ (that is, with clear signs of AGN activity, first part of Table 3), we find typical $\mathrm{BH}$ masses of $3 \times 10^{7} M_{\odot}$, ranging from $6 \times 10^{6} M_{\odot}$ to $3 \times 10^{8} M_{\odot}$. The estimated $\mathrm{BH}$ masses for those LIRGs classified as composite or $\mathrm{H}$ II are also in this range. The masses of the LIRG BHs appear to be similar to those of the currently growing $\mathrm{BHs}$ in the local universe hosted in late-type galaxies (Heckman et al. 2004; Schawinski et al. 2010).

The typical Eddington ratios $\left(L_{\mathrm{bol}}(\mathrm{AGN}) / L_{\mathrm{Edd}}\right)$ for the LIRGs with an estimate of $M_{\mathrm{BH}}$ are $2 \times 10^{-2}$, and range between $5 \times 10^{-4}$ and 0.1 . This means that the BHs in local LIRGs are accreting at a lower efficiency than those in local ULIRGs (typical values of 0.08-0.4 depending on the method used for estimating the BH mass; Dasyra et al. 2006; Veilleux et al. 2009).

The majority of LIRGs with spectroscopically resolved [Ne III] $15.56 \mu \mathrm{m}$ lines are classified as Seyfert galaxies. However, in local LIRGs there is a high fraction of composite objects (AGN/starburst). Moreover, this fraction remains approximately constant as a function of IR luminosity from galaxies with $L_{\mathrm{IR}} \sim 10^{10} L_{\odot}$ up to ULIRGs (Yuan et al. 2010). As can be seen from Figure 1, composite nuclei in local LIRGs have values for the FWHM of [ $\mathrm{Ne}$ III] $15.56 \mu \mathrm{m}$, lower on average than those of Seyfert nuclei but higher than those of nuclei classified as $\mathrm{H}$ II 
and the instrumental resolution. Assuming that these composite nuclei do indeed host an AGN and that the [Ne III] $15.56 \mu \mathrm{m}$ line is probing their NLR, then their BH masses ought to be less than $\sim 2-3 \times 10^{7} M_{\odot}$. The estimated typical AGN bolometric luminosities from the mid-IR spectral decomposition are $10^{43} \mathrm{erg} \mathrm{s}^{-1}$ or less, so we cannot constrain their Eddington ratios.

The BHs of local LIRGs are only marginally less massive than those of local ULIRGs. The $\mathrm{BH}$ masses of the latter are in the range $\sim 10^{7}-5 \times 10^{8} M_{\odot}$ (Tacconi et al. 2002; Dasyra et al. 2006; Veilleux et al. 2009). Samples of local ULIRGs are mostly dominated by gas-rich interacting galaxies and major mergers (Sanders \& Mirabel 1996), with coalesced ULIRGs having slightly larger BH masses than pre-coalescence ULIRGs (Dasyra et al. 2006). Our sample is both flux and volume-limited, and thus is composed mostly of LIRGs with $L_{\mathrm{IR}} \sim 1-2 \times 10^{11} L_{\odot}$ (individual galaxies), which also tend to be spiral galaxies, minor mergers, and galaxies in groups. That is, morphologically local LIRGs are not dominated in numbers by major mergers (see, e.g., Sanders \& Ishida 2004; Kaviraj 2009; Pereira-Santaella 2012) and their BH masses are similar to those of pre-coalescent ULIRGs (Dasyra et al. 2006).

\subsection{Star Formation Rates}

We computed the SFRs for our sample of LIRGs using a number of IR-based indicators, including the fine-structure [Ne II] $12.81 \mu \mathrm{m}$ line (Roche et al. 1991; Ho \& Keto 2007), the $11.3 \mu \mathrm{m}$ PAH feature (Brandl et al. 2006; Diamond-Stanic \& Rieke 2012), and the total 8-1000 $\mu \mathrm{m}$ IR luminosity (Kennicutt 1998). Although it is not clear whether the presence of an AGN could destroy the PAH carriers or not, at the typical luminosities of the AGN hosted in local LIRGs, the $11.3 \mu \mathrm{m}$ PAHs do not seem to be affected (Diamond-Stanic \& Rieke 2010).

For the integrated SFRs, we used $L_{\mathrm{IR}}$ of the individual galaxies as given in Alonso-Herrero et al. (2012, their Table 1) after subtracting the AGN contribution. We used the Kennicutt (1998) relation in terms of the IR luminosity converted to a Kroupa IMF:

$$
\operatorname{SFR}\left(M_{\odot} \mathrm{yr}^{-1}\right)=1.14 \times 10^{-10}\left(L_{\mathrm{IR}}, L_{\odot}\right) .
$$

We obtained total SFRs for the individual galaxies in our sample of between 1 and $60 M_{\odot} \mathrm{yr}^{-1}$.

For the nuclear SFR, we used the Spitzer/IRS spectroscopy. At the median distance of $65 \mathrm{Mpc}$ for our sample of LIRGs, the IRS SH slit width (4.7 arcsec) subtends typically $1.5 \mathrm{kpc}$. We use the following recipes put forward by Diamond-Stanic \& Rieke (2012) to compute the nuclear SFRs based on the [Ne II] $12.81 \mu \mathrm{m}$ line $11.3 \mu \mathrm{m}$ PAH feature luminosities:

$$
\begin{gathered}
\operatorname{SFR}\left(M_{\odot} \mathrm{yr}^{-1}\right)=9.6 \times 10^{-9} L\left(11.3 \mu \mathrm{m}, L_{\odot}\right) \\
\operatorname{SFR}\left(M_{\odot} \mathrm{yr}^{-1}\right)=8.9 \times 10^{-8} L\left([\mathrm{Ne} \mathrm{II}], L_{\odot}\right) .
\end{gathered}
$$

Diamond-Stanic \& Rieke (2012) calibrated Equations (5) and (6) for galaxies with $L_{\mathrm{IR}}<10^{11} L_{\odot}$ using the Rieke et al. (2009) templates and a Kroupa IMF. We choose to use these calibrations rather than those for higher IR luminosities because the median value of the IR luminosity of the individual galaxies in our sample is $1.3 \times 10^{11} L_{\odot}$. In addition, the width of the IRS $\mathrm{SH}$ slit only probes the nuclear regions of our sample of LIRGs and thus lower IR luminosities.

We also note that Equation (5) assumed that the flux of the $11.3 \mu \mathrm{m}$ PAH feature was measured using PAHFIT (Smith et al. 2007). The spectral coverage $(\sim 10-18 \mu \mathrm{m})$ and spectral resolution of the $\mathrm{SH}$ data used here are not adequate to use PAHFIT. We measured the PAH fluxes using a local continuum (Section 2 and Table 2). Following Smith et al. (2007), we applied a multiplicative factor of two to the PAH fluxes measured with a local continuum to make a proper comparison with PAHFIT fluxes. We also corrected the $11.3 \mu \mathrm{m}$ PAH fluxes for extinction using the nuclear strength of the $10 \mu \mathrm{m}$ silicate feature (from Alonso-Herrero et al. 2012) and the extinction law given in Smith et al. (2007). For most of our local LIRGs, this correction is small, as the silicate absorptions are moderate (see, e.g., Figure 6 in Alonso-Herrero et al. 2012) as compared with the more deeply embedded population of local ULIRGs (Spoon et al. 2007).

While the [Ne II] $12.81 \mu \mathrm{m}$ emission in low-luminosity and moderate-luminosity AGNs is mostly produced by SF, in highluminosity AGNs, this emission line can also have an important contribution from the AGNs (Pereira-Santaella et al. 2010b). To correct the [Ne II] $12.81 \mu \mathrm{m}$ line flux for any possible AGN contribution, we followed the method put forward by Veilleux et al. (2009). This method reproduces the observed values of the [O IV] $25.89 \mu \mathrm{m} /[\mathrm{Ne}$ II] $12.81 \mu \mathrm{m}$ line ratio (given in Alonso-Herrero et al. 2012) as a fractional combination of the typical line ratio of an AGN ( 4) and that of a starburst galaxy $(\sim 0.01)$, where the AGN contribution is the free parameter. Using this method, we find that the AGN contribution to the nuclear [Ne II] $12.81 \mu \mathrm{m}$ emission of local LIRGs is small. These corrections are only necessary for 10 LIRGs and the AGN contribution to the nuclear [Ne II] $12.81 \mu$ m fluxes ranges from $3 \%$ to $53 \%$ (average $\sim 18 \%$ ).

Figure 4 (left panel) compares the nuclear SFRs as derived from the [Ne II] $12.81 \mu \mathrm{m}$ line (after removing the AGN contribution) and from the $11.3 \mu \mathrm{m}$ PAH feature. We have color coded the symbols by the AGN bolometric contribution to IR luminosity of the galaxies (see Alonso-Herrero et al. 2012). As can be seen from this figure, the majority of LIRGs with small or no $(<25 \%)$ AGN contribution show a good agreement between the derived nuclear SFRs using the two indicators. Indeed, we find that the ratio between the nuclear SFR estimated from the $11.3 \mu \mathrm{m}$ PAH feature and that from the [Ne II] $12.81 \mu \mathrm{m}$ line is on average $1.0 \pm 0.3$.

In a few cases with a significant AGN bolometric contribution, the nuclear SFR derived from the [Ne II] $12.81 \mu \mathrm{m}$ line is higher than that from the $11.3 \mu \mathrm{m}$ PAH feature, even after correcting for the AGN contribution. Given the good agreement between the nuclear SFR computed with the two methods for LIRGs with small or no AGN contribution and to avoid AGN contamination issues from now on for the nuclear SFR, we use those derived from the $11.3 \mu \mathrm{m}$ PAH feature. Thus, the individual nuclei of the sample of local LIRGs show values of the nuclear SFR of between $\sim 0.8$ and $\sim 20 M_{\odot} \mathrm{yr}^{-1}$.

In Figure 4 (right), we compare the total SFR against the nuclear (typically on scales of $1.5 \mathrm{kpc}$ ) SFR for the individual galaxies of the LIRG sample. It is clear that a significant fraction of the individual galaxies in our sample have more than half of their total SFRs taking place outside of the nuclear (1-2 kpc) regions. For the individual galaxies of the sample, the average value of the nuclear SFR over the total SFR ratio is $0.5 \pm 0.3$. This agrees with findings using other indicators (Hattori et al. 2004; Alonso-Herrero et al. 2006; Díaz-Santos et al. 2010; Rodríguez-Zaurín et al. 2011). We also note, however, that in approximately $20 \%$ of our volume-limited sample of LIRGs, the nuclear emission accounts for most of the total SFR measured 

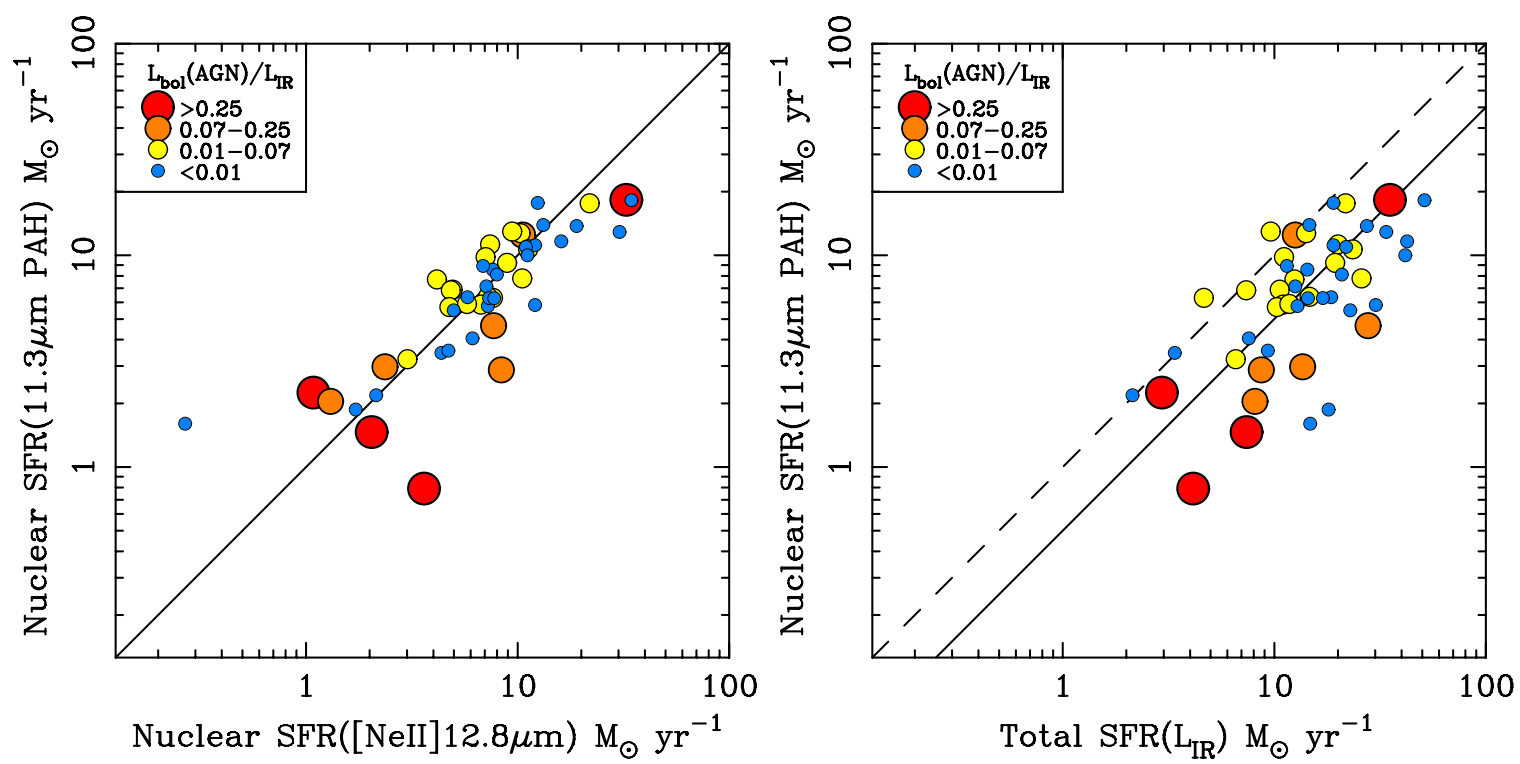

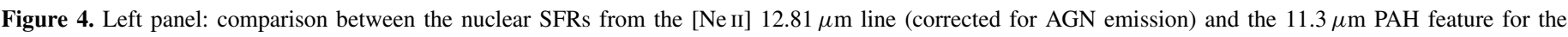

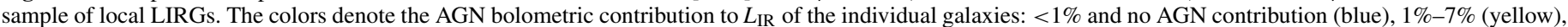

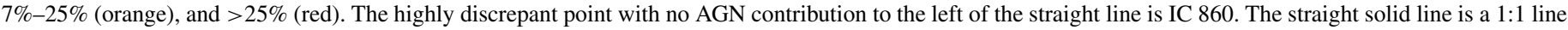

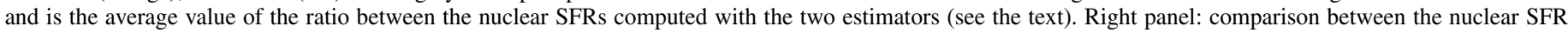

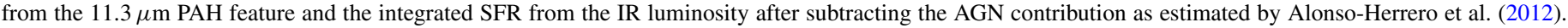

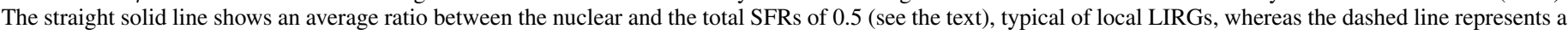
1:1 relation.

(A color version of this figure is available in the online journal.)

in the individual galaxies, as is the case for a large fraction of local ULIRGs.

Incidentally, the same comparison for the nuclear SFRs (Figure 4, left) but using the SFR equations calibrated for $L_{\mathrm{IR}}>10^{11} L_{\odot}$ (see Diamond-Stanic \& Rieke 2012) results in nuclear SFRs from the $11.3 \mu \mathrm{m}$ PAH feature that are on average a factor of 1.3 higher than those from the [Ne II] $12.81 \mu \mathrm{m}$ line. Moreover, the ratio between the nuclear SFR from the $11.3 \mu \mathrm{m}$ PAH feature and the total SFR from $L_{\mathrm{IR}}$ would also be on average 1.3. This gives us confidence that we used the appropriate SFR calibrations for the nuclear rates.

\subsection{Relation between BH Accretion Rates and SFR in LIRGs}

The presence of an AGN is an unambiguous signpost of a period of $\mathrm{BH}$ growth. The AGN luminosity can then be expressed in terms of the BHAR $\dot{m}_{\mathrm{BH}}$ and the mass-energy conversion efficiency $\epsilon$ (see Alexander \& Hickox 2012 and references therein):

$$
\dot{m}_{\mathrm{BH}}\left(M_{\odot} \mathrm{yr}^{-1}\right)=0.15(0.1 / \epsilon)\left(L_{\mathrm{bol}} / 10^{45} \mathrm{erg} \mathrm{s}^{-1}\right) .
$$

We used this relation with the typical value of $\epsilon=0.1$ in the local universe (Marconi et al. 2004) and $L_{\text {bol }}(A G N)$ from the mid-IR spectral decomposition (see Alonso-Herrero et al. 2012). We obtained BHAR between 0.0007 and $0.08 M_{\odot} \mathrm{yr}^{-1}$ for local LIRGs. The uncertainties of the estimates of the BHAR are dominated by the uncertainties of the AGN bolometric luminosities that are typically less than 0.4 dex (see Alonso-Herrero et al. 2012, for details).

Having calculated the BHAR, we can now compare them with the nuclear and total SFR for the sample of LIRGs. The distribution of SFR $/ \dot{m}_{\mathrm{BH}}$ can provide clues as to whether AGN and ongoing SF activity are contemporaneous in local LIRGs. Recent numerical simulations by Hopkins (2012) predict a time offset between the peaks of these activities that is thought to depend on the physical scale within the galaxy and the dynamical time of the galaxy, among other parameters.

Figure 5 shows the distribution of SFR $/ \dot{m}_{\mathrm{BH}}$ for the sample of local LIRGs for the nuclear $(\sim 1.5 \mathrm{kpc})$ and the integrated SFRs, only for those LIRGs with an estimate of $L_{\text {bol }}$ (AGN). The nuclear and integrated $\mathrm{SFR} / \dot{m}_{\mathrm{BH}}$ are in the range $\sim 12$ to $\sim 2 \times 10^{4}$, with median values of the nuclear and integrated $\log \left(\mathrm{SFR} / \dot{m}_{\mathrm{BH}}\right)$ of 3.1 and 3.4 , respectively. Most of the nuclei classified as Seyfert from optical spectroscopy show nuclear $\log \left(\mathrm{SFR} / \dot{m}_{\mathrm{BH}}\right)<3.0$. The nuclei classified as composite tend to show larger values of the nuclear SFR to BHAR ratio, as expected if they were powered by both SB and AGN activity.

For the entire sample of local LIRGs, we obtain $\sum \mathrm{SFR} / \sum \dot{m}_{\mathrm{BH}} \geqslant 2900$. This is a lower limit because for half of the sample, we did not detect an AGN component and therefore we are only using an upper limit for their BHAR. If we repeat this only for those LIRGs with an estimate of their AGN bolometric luminosities, then we obtain a time-averaged value of $\mathrm{SFR} / \dot{m}_{\mathrm{BH}} \simeq 2500$, if we assume that all LIRGs go through an AGN phase, that is, a $\sim 50 \%$ duty cycle. The two values are consistent with each other taking into account the typical uncertainties in calculating $L_{\text {bol }}(\mathrm{AGN})(\sim 0.4 \mathrm{dex}$; Alonso-Herrero et al. 2012). This ratio is a few times higher than that measured for the local population of bulge-dominated galaxies $\left(\sim 10^{3}\right.$, Heckman et al. 2004) and the local normalization of the $\mathrm{BH}$ mass versus bulge mass relation (Marconi \& Hunt 2003; Häring $\&$ Rix 2004). Even though the SFRs of LIRGs are much higher than those of local bulge galaxies, the time-averaged SFR $/ \dot{m}_{\mathrm{BH}}$ value of local LIRGs is similar to that of local bulge galaxies during the first 0.3 Gyr of their ongoing starburst (Wild et al. 2010, their Figure 9). This suggests the existence of similar time delays between the peak of SF and BH growth in local LIRGs as well. 

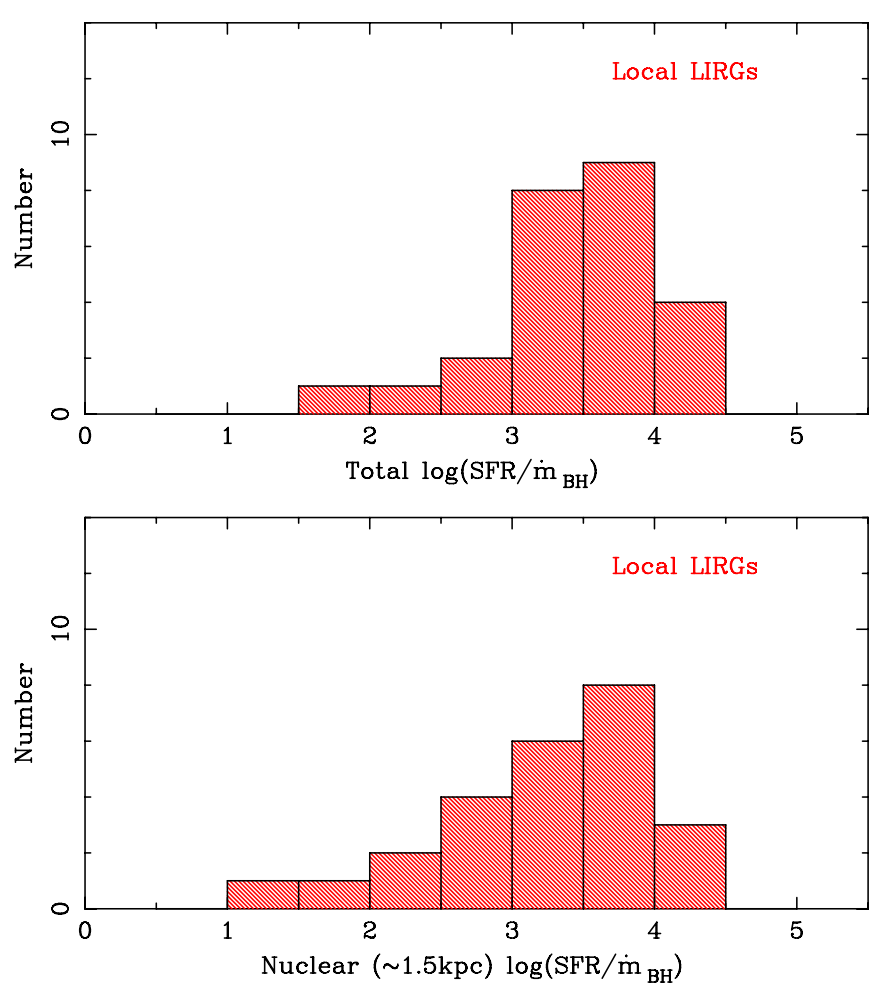

Figure 5. Distributions of the $\mathrm{SFR} / \dot{m}_{\mathrm{BH}}$ ratios for nuclear (typical scales of $1.5 \mathrm{kpc}$ ) SFRs (lower panel) and integrated SFRs (upper panel) for the sample of local LIRGs with an estimate of the AGN luminosity. The nuclear SFR is from the $11.3 \mu \mathrm{m}$ PAH feature and the integrated SFR is from $L_{\mathrm{IR}}$ after subtracting the AGN component (see the text for details).

(A color version of this figure is available in the online journal.)

\section{DISCUSSION}

\subsection{Comparison between the AGNs in LIRGs and the Optically Identified RSA Seyferts}

In this section, we compare the properties of the AGNs identified in local LIRGs with the optically selected Seyfert galaxies in the revised-Shapley-Ames (RSA) catalog (Sandage \& Tammann 1987; Maiolino \& Rieke 1995). This optical sample was spectroscopically selected from the original galaxymagnitude-limited sample of RSA galaxies and is believed not to be biased against low-luminosity Seyfert galaxies. Also, as for the sample of local LIRGs studied in this work, the Seyfert activity in the RSA sample does not appear to be driven primarily by mergers for the majority of the galaxies. Finally, the AGN bolometric luminosities of the RSA Seyferts are similar to those of the local LIRGs.

The median values of SFR $/ \dot{m}_{\mathrm{BH}}$ for our LIRGs are on average higher than those of local Seyfert galaxies, especially the nuclear values (see Figure 5 and Diamond-Stanic \& Rieke 2012). Of course, this is not completely unexpected as local LIRGs, including those hosting an AGN, are essentially selected by their SF activity, whereas the RSA Seyferts are selected by their host galaxy magnitudes. The latter is more likely probing the stellar mass, rather than the SF activity.

We can make a more meaningful comparison if we identify the IR-bright Seyferts in the RSA sample. We set the limit to $\log \left(\mathrm{L}_{\mathrm{IR}} / L_{\odot}\right)=10.8$ to match approximately the lower limit covered by the IR luminosities of the individual galaxies of our sample of local LIRGs (see Table 1 in Alonso-Herrero et al. 2012). We also included from our sample the newly
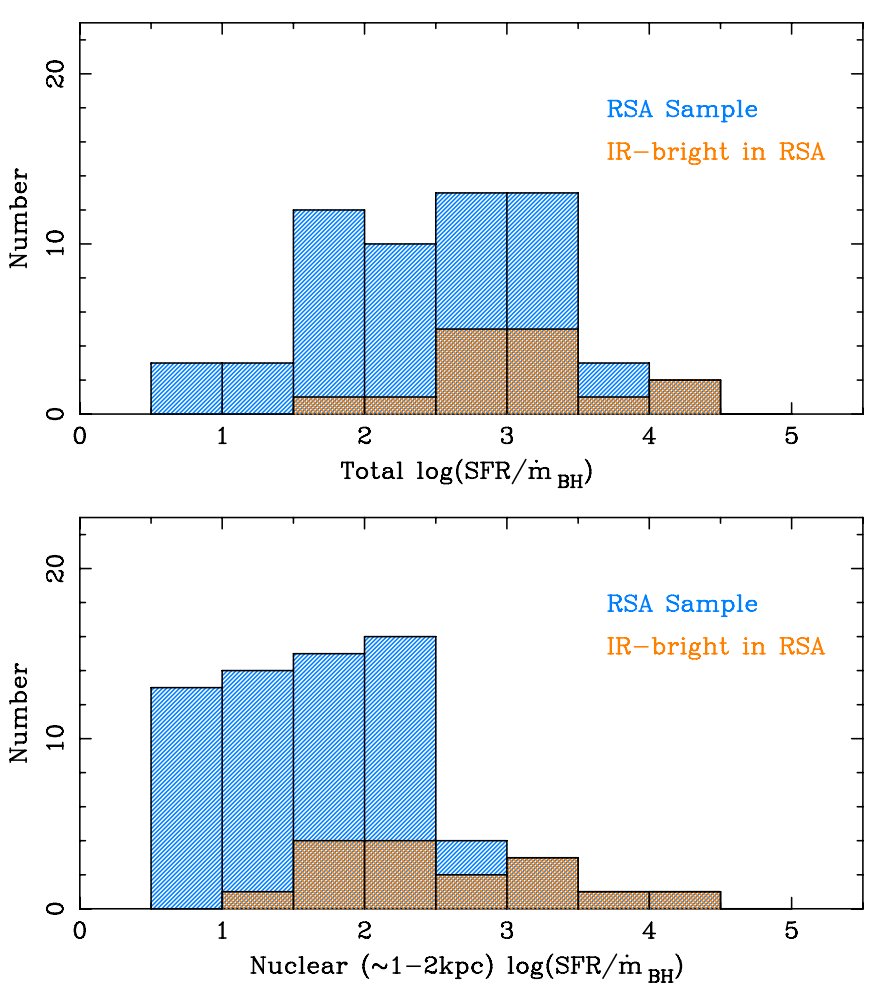

Figure 6. Distributions of the ratio between the nuclear SFR and the BHAR (bottom panel) and the integrated SFR and the BHAR (upper panel) for the RSA sample of AGNs (blue). In orange, we mark those RSA AGNs that are also IR-bright galaxies (see the text). We only included RSA galaxies with $\dot{m}_{\mathrm{BH}}>10^{-4} M_{\odot} \mathrm{yr}^{-1}$.

(A color version of this figure is available in the online journal.)

identified Seyfert nuclei with an estimate of $L_{\text {bol }}(A G N)$. The values of $\mathrm{SFR} / \dot{m}_{\mathrm{BH}}$ for the RSA Seyferts are taken from Diamond-Stanic \& Rieke (2012), except for those already included in our sample of LIRGs. Diamond-Stanic \& Rieke (2012) computed the nuclear SFRs from the $11.3 \mu \mathrm{m}$ PAH feature and the MIPS $24 \mu \mathrm{m}$ photometry, and $L_{\text {bol }}(\mathrm{AGN})$ from the [O IV] $25.89 \mu \mathrm{m}$ line. Finally, for the few RSA Seyferts deemed to have their [O IV] $25.89 \mu \mathrm{m}$ luminosities strongly contaminated by SF, we estimated $L_{\text {bol }}(\mathrm{AGN}$ ) (and thus BHAR) from their hard X-ray luminosities and using a bolometric correction. In this comparison, we only included RSA Seyferts with $\dot{m}_{\mathrm{BH}}>10^{-4} M_{\odot} \mathrm{yr}^{-1}$ to match the approximate AGN detection threshold in the LIRG sample. With this limit, the IR-bright galaxy fraction in the RSA sample of Seyfert galaxies is then $\sim 22 \% \pm 5 \%$.

Figure 6 shows the distributions of the nuclear and total $\mathrm{SFR} / \dot{m}_{\mathrm{BH}}$ for all of the RSA Seyferts, indicating which ones are also IR bright. Clearly, those RSA Seyferts classified as IR bright have ratios similar to, although slightly smaller than, those of the complete sample of local LIRGs. Given the fact that the AGNs in the RSA sample are not very different from those in the LIRGs, the results above would suggest that the bright AGN phase comes after and is somewhat distinct from the LIRG star-forming phase. This interpretation would be consistent with observational works showing a delay between the onset of the SF activity and the latter feeding of the AGN with the consequent BH growth (Davies et al. 2007; Wild et al. 2010) as well as with predictions from numerical simulations (e.g., Hopkins 2012). 


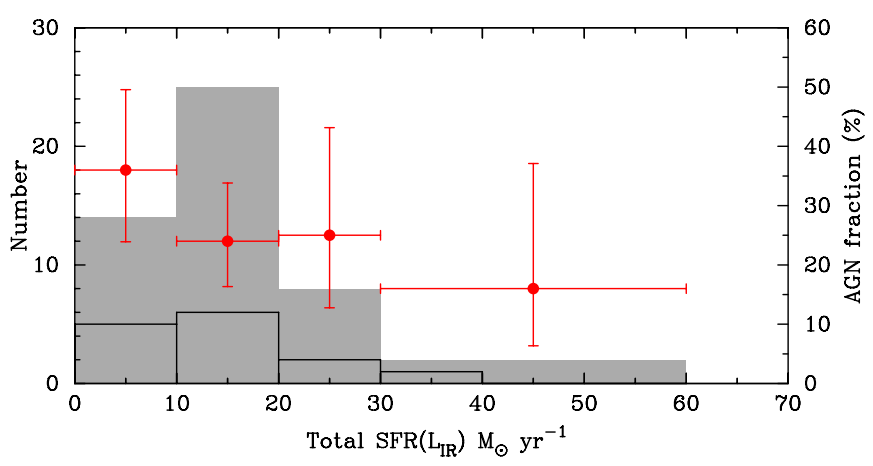

Figure 7. Distributions of the integrated SFRs of the local LIRGs (gray histograms) and of those with a Seyfert classification and/or [ $\mathrm{Ne} \mathrm{v]} \mathrm{emitters}$ (solid line histograms). The data points represent the AGN fraction (scale on the right-hand vertical axis) in SFR intervals, with $1 \sigma$ error bars for the AGN fraction.

(A color version of this figure is available in the online journal.)

\subsection{SFRs versus AGN Detection in Local LIRGs}

It is well known that the AGN fraction increases with increasing IR luminosity from LIRGs to ULIRGs, and at the highest IR luminosities the AGN might dominate bolometrically the luminosity of the system (Veilleux et al. 1995, 2009; Yuan et al. 2010; Nardini et al. 2010; Alonso-Herrero et al. 2012). We now examine further the possibility that the IR-bright AGN phase comes after the distinct IR-bright star-forming phase. If that were the case, then we would expect the AGN fraction in LIRGs to be higher at the lowest SFR. The AGN fraction here is computed only for those LIRGs with a secure AGN detection, that is, a Seyfert classification and/or the presence of mid-IR [ $\mathrm{Ne} \mathrm{v}]$ lines. As in the previous section, we computed the integrated SFR of the individual galaxies from $L_{\mathrm{IR}}$ after subtracting the AGN contribution.

Figure 7 shows the AGN fraction as a function of the total SFR of the galaxy. There is a tendency for LIRGs in lowest SFR bin to have a higher AGN incidence $\left(36 \%_{-12 \%}^{+14 \%}\right)$ than those in the highest SFR bin $\left(17 \%_{-11 \%}^{+20 \%}\right)$. Alternatively, this could be explained if it is harder to detect AGN in LIRGs with very high SFRs, especially if it takes place mostly in the nuclear regions. Conversely, it may be easier to identify an AGN for low nuclear SFRs and extended SF. The first explanation is not likely, however, as most of the LIRGs in our sample in the high SFR bin also tend to have rather extended SF. In other words, from Figure 4 (right panel), there is no tendency for the fraction of nuclear SFR to increase with the total SFR of the galaxy. In fact, in local LIRGs the mid-IR emission (both from AGN and SF activity) only starts to be highly concentrated in the central regions for IR luminosities above $10^{11.8} L_{\odot}$, as shown by Díaz-Santos et al. (2010). We conclude that the higher AGN incidence at low SFRs in local LIRGs provides further evidence for a time delay between the peaks of the SF and $\mathrm{BH}$ growth.

\subsection{Key Differences between Merger and Non-merger Local LIRGs}

Before we explore the role of the LIRG phase in the context of SF activity and $\mathrm{BH}$ growth of massive late-type galaxies (next section), it is important to point out the differences in the SF histories of merger and non-merger LIRGs in the local universe.

The triggering of the activity in local ULIRGs, and likely also in the most luminous local LIRGs, is driven by major mergers. As a consequence, ULIRGs tend to host more luminous
AGNs (quasar or nearly quasar-like luminosities), slightly more massive BHs, and have higher AGN bolometric contributions than local LIRGs (see Section 3.1 and Veilleux et al. 2009; Nardini et al. 2010; Alonso-Herrero et al. 2012). Local merger LIRGs do show evidence for a recent and intense period of SF that consumed the gas faster, that is, a bursty SFR (see, e.g., Alonso-Herrero et al. 2000, 2001) as in local ULIRGs (Rodríguez-Zaurín et al. 2010). In this respect, most of the local merger-LIRGs could be considered as sub-ULIRGs, and they might go through a future ULIRG phase or may already have experienced such a phase (Murphy et al. 2001).

Major mergers, however, do not dominate in numbers the population of local LIRGs, especially at $L_{\mathrm{IR}}<3 \times 10^{11} L_{\odot}$, as inferred from morphological studies (see, e.g., Sanders \& Ishida 2004; Alonso-Herrero et al. 2006; Kaviraj 2009). Therefore, in most local LIRGs, the current episodes of SF activity and $\mathrm{BH}$ growth are likely the result of a less violent process, e.g., via minor mergers, fly-by companions, and/or secular evolution. Poggianti \& Wu (2000) showed that most isolated galaxies in their sample of IR-bright galaxies showed on average more moderate optical Balmer absorption features than the strongly interacting systems in their sample. This suggests that in non-merger LIRGs there is not a strong contribution from a dominant post-starburst stellar population that would be associated with a recent bursty SF history. This agrees with the finding that most of the mass in non-merger LIRGs is from evolved stellar populations formed a few Gyr ago with a much smaller contribution in mass from a relatively young stellar population (Kaviraj 2009; Alonso-Herrero et al. 2006, 2010).

\subsection{The Role of the LIRG Phase in the Growth of $B H$ at $L_{\mathrm{IR}}<3 \times 10^{11} L_{\odot}$}

The cartoon in Figure 8 illustrates our proposed scenario for the current episode of $\mathrm{BH}$ growth in a non-merger local LIRG typically with $L_{\mathrm{IR}}<3 \times 10^{11} L_{\odot}$ (i.e., integrated $\mathrm{SFR}<30 M_{\odot} \mathrm{yr}^{-1}$ ). This IR luminosity marks approximately the border between local populations of LIRGs dominated by major merger and populations of disky and relatively isolated LIRGs (Sanders \& Ishida 2004).

We assume that the galaxy has an old stellar population formed a few Gyr ago (Kaviraj 2009; Alonso-Herrero et al. 2010) and an existing BH. Since the galaxy did not experience a recent major merger but rather a less violent process that induced the last episode of SF, we can assume an exponentially decaying $\operatorname{SFR}$ for it, as $\operatorname{SFR}(t)=\operatorname{SFR}_{0} \mathrm{e}^{-t / \tau}$, where $\tau$ is the $e$-folding time. The peak $\operatorname{SFR}(t=0) \simeq 30 M_{\odot} \mathrm{yr}^{-1}$ is driven by the $L_{\mathrm{IR}}$ limit set above. From the position of local AGN on the color-magnitude diagram, Schawinski et al. (2009) demonstrated that the $e$-folding time cannot be too short (a few Myr or bursty) or too long (i.e., constant SFR). The high AGN detection rate in local LIRGs (Veilleux et al. 1995; Yuan et al. 2010; Alonso-Herrero et al. 2012) implies that LIRGs are able to feed the BH (i.e., show AGN activity) roughly during half of the LIRG phase (defined as having integrated SFR $>10 M_{\odot} \mathrm{yr}^{-1}$, see Figure 4). Moreover, given the moderate BH masses inferred for LIRGs (Section 3.1), this AGN activity is likely to be sustained for $\sim 5 \times 10^{8} \mathrm{yr}$, that is, the typical lifetime of the $\mathrm{BH}$ growth for $M_{\mathrm{BH}}<10^{8} M_{\odot}$ (see Marconi et al. 2004).

In this simple scenario, the galaxy would be first classified optically as an H II LIRG and would show elevated integrated and nuclear SFRs. Provided that there is a mechanism to bring the gas near the $\mathrm{BH}$ gravitational potential, after a time the existing $\mathrm{BH}$ starts accreting material at a sufficiently high rate. 


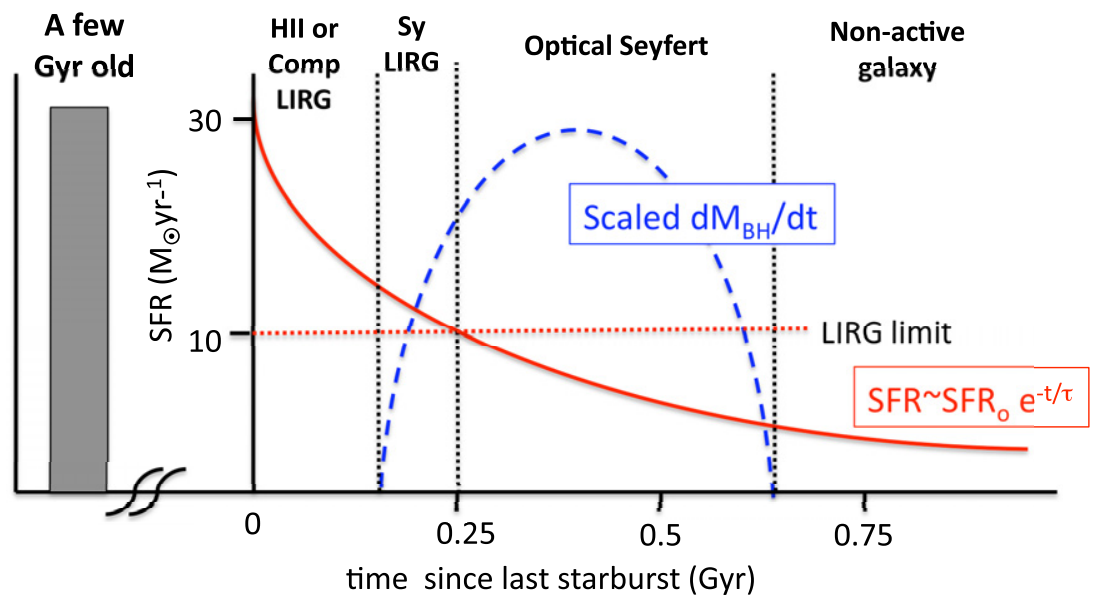

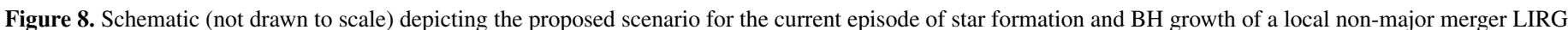

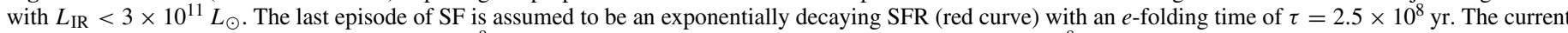

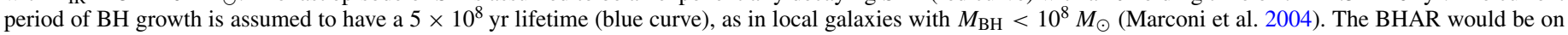
average $\sim 3000$ times lower than the SFR during the IR-bright phase.

(A color version of this figure is available in the online journal.)

Then, the galaxy would still have a Seyfert-like AGN luminosity. Because there is an elevated nuclear SFR, the galaxy would be optically classified first as composite (AGN/SB) and later on as a Seyfert LIRG as the SFR decreases. In both cases, the $\mathrm{SFR} / \dot{m}_{\mathrm{BH}}$ ratios would be on average higher than those of optically identified Seyferts. After a few hundred million years, the SFR would be below $10 M_{\odot} \mathrm{yr}^{-1}$ and thus it would not be classified as a LIRG any longer. However, the AGN phase may still be active for another few hundred million years as Seyfertlike AGNs do not require large amounts of gas to remain active (Hopkins 2012). At this point, the galaxy would be identified as an optical Seyfert with a lower value of the SFR/BHAR ratio and a moderate SFR. Eventually, both the SF and the BHAR would be low and the galaxy may not be identified as a Seyfert. An SFR $e$-folding time of $\tau \sim 2.5 \times 10^{8} \mathrm{yr}$ and a BH growth lifetime of $\sim 5 \times 10^{8} \mathrm{yr}$ would reproduce the SFRs as well as the Seyfert and composite fractions of local LIRGs (Yuan et al. 2010).

In summary, in the local universe, LIRGs with $L_{\mathrm{IR}}<$ $3 \times 10^{11} L_{\odot}$ LIRGs and an AGN represent an early phase of the (possibly episodic) growth of BHs in massive spiral galaxies with high SFR, not necessarily associated with a major merger event. An H II-like LIRG phase would predate the AGN classified LIRG phase. The latter in turn would be followed by an optically identified AGN phase where the galaxy still shows an appreciable SFR, but below the LIRG limit. Although the triggering mechanisms for the SB and AGN activity of LIRGs and ULIRGs might be different, it appears that the sequence of events is similar. Namely, ULIRGs are believed to involve the interaction between two gas-rich galaxies that results first in a high SFR and then an optical QSO phase (see Sanders et al. 1988; Sanders \& Mirabel 1996; Yuan et al. 2010 and references therein). The end product, in contrast, is rather different, with ULIRGs probably evolving into moderate-mass ellipticals (Sanders \& Mirabel 1996; Dasyra et al. 2006; Hopkins et al. 2008) and non-merger LIRGs likely remaining late-type galaxies.

\section{SUMMARY}

We have studied the current co-evolution of the SF activity and $\mathrm{BH}$ growth for a complete sample of 51 local LIRGs (see
Alonso-Herrero et al. 2006, 2012, for details on the sample). We used Spitzer/IRS SH $(R \sim 600)$ data in the $\sim 10-18 \mu \mathrm{m}$ spectral range to estimate $\mathrm{BH}$ masses from resolved [Ne III] $15.56 \mu \mathrm{m}$ fine-structure lines, and to measure nuclear SFRs from the $11.3 \mu \mathrm{m}$ PAH feature and the [Ne II] $12.81 \mu \mathrm{m}$ line.

We detected spectrally resolved [Ne III] $15.56 \mu \mathrm{m}$ lines in 19 LIRGs, with gas velocity dispersions between 102 and $368 \mathrm{~km} \mathrm{~s}^{-1}$. Most of these LIRGs are classified as Seyferts and/or are [Ne v] emitters. We assumed that [Ne III] $15.56 \mu \mathrm{m}$ probes gas arising from the NLR in the AGN LIRGs and then used the Dasyra et al. (2011) relation between $M_{\mathrm{BH}}$ and [Ne III] $15.56 \mu \mathrm{m}$ velocity dispersion. We combined gas velocity dispersions from [Ne III] $15.56 \mu \mathrm{m}$ and [O III] $\lambda 5007$ and stellar velocity dispersions from the literature to obtain $\mathrm{BH}$ masses between $M_{\mathrm{BH}}=6 \times 10^{6} M_{\odot}$ and $M_{\mathrm{BH}}=3.5 \times 10^{8} M_{\odot}$ (median value of $3 \times 10^{7} M_{\odot}$ ). The derived AGN bolometric luminosities of $L_{\mathrm{bol}}(\mathrm{AGN})=(0.4-50) \times 10^{43} \mathrm{erg} \mathrm{s}^{-1}$ (median of $\sim 1.4 \times 10^{43} \mathrm{erg} \mathrm{s}^{-1}$; see Alonso-Herrero et al. 2012) imply a BHAR of $\dot{m}_{\mathrm{BH}} \simeq 0.0007-0.08 M_{\odot} \mathrm{yr}^{-1}$ and relatively low Eddington ratios (typically $2 \times 10^{-2}$ ).

Although AGNs accompany the SF activity in the large majority of local LIRGs (Veilleux et al. 1995; Yuan et al. 2010; Alonso-Herrero et al. 2012), in most cases the AGN is not energetically dominant (Alonso-Herrero et al. 2012). Using the $11.3 \mu \mathrm{m}$ PAH feature, we obtained nuclear (on typical scales of $1.5 \mathrm{kpc}$ ) SFRs for the individual galaxies of between $\sim 0.8$ and $\sim 20 M_{\odot} \mathrm{yr}^{-1}$ and using $L_{\mathrm{IR}}$-integrated SFRs between 1 and $60 M_{\odot} \mathrm{yr}^{-1}$ (for a Kroupa IMF). In a large fraction of local LIRGs, this ongoing SF activity is not only taking place in the nuclear regions but it is also spread out on kpc scales throughout the galaxy. From the IR estimates in this work, the median value for the sample of local LIRGs is SFR(nuclear $\sim 1.5 \mathrm{kpc}$ )/ $\mathrm{SFR}($ total $)=0.5$.

We found that local LIRGs have a time-averaged value of $\mathrm{SFR} / \dot{m}_{\mathrm{BH}} \geqslant 2900$, which is a few times higher than that of the local population of bulge-dominated galaxies $\left(\sim 10^{3}\right.$, Heckman et al. 2004) and the local normalization of the BH mass versus bulge mass relation (Marconi \& Hunt 2003; Häring \& Rix 2004). However, this ratio is similar to the value of local bulge galaxies averaged during the first $\sim 0.3 \mathrm{Gyr}$ of the last starburst (Wild et al. 2010), that is, before the peak of the BH growth. 
Local LIRGs have on average higher SFR $/ \dot{m}_{\mathrm{BH}}$ ratios (both nuclear and total SFR) than the optically selected RSA Seyferts in the local universe. However, the IR-bright RSA Seyferts have similar ratios to those of local LIRGs. Since the overall properties of the AGNs in local LIRGs and in the RSA sample are very similar, we concluded that the AGN phase comes after and is somewhat distinct from the LIRG star-forming phase. The AGN fraction in local LIRGs appears to be a function of the integrated SFR, with LIRGs in the low SFR bin having a higher AGN (i.e., Seyfert) incidence than those with high SFRs. This provides further support to the scenario that in local LIRGs, the peak of intense IR-bright SF activity occurs prior to the AGN phase.

Finally, we put forward a simple model to explore the role of the LIRG phase in the last episode of BH growth at $L_{\mathrm{IR}}<3 \times 10^{11} L_{\odot}$. At these luminosities, most LIRGs are not major mergers, and have an old stellar population formed a few Gyr ago and a current episode of SF. The latter are only a small mass fraction of the total stellar mass. We propose that an exponentially decaying SFR with a peak $\operatorname{SFR}(t=0) \simeq$ $30 M_{\odot} \mathrm{yr}^{-1}$ and an $e$-folding time of $\tau \sim 2.5 \times 10^{8} \mathrm{yr}$ together with a $5 \times 10^{8} \mathrm{yr}$ period of $\mathrm{BH}$ growth would explain the observed SFRs and fractions of Seyfert and composite objects in local LIRGs. In this scenario, non-merger LIRGs hosting an AGN represent an early phase of the possibly episodic growth of the BH which is followed by an optically Seyfert phase in massive galaxies with relatively high SFR in their host galaxies.

A.A.-H. thanks the Astrophysics Department of the University of Oxford, where most of this work was conducted, for their warm hospitality. This work was supported in part by the Spanish Plan Nacional de Astronomía y Astrofísica under grants AYA2009-05705-E and AYA2010-21161-C02-1 and by the Augusto González Linares Program from the Universidad de Cantabria. M.P.-S. is funded by an ASI fellowship under contract I/005/11/0. The authors thank the referee for comments that helped improve the paper. A.M.D. acknowledges support from the Southern California Center for Galaxy Evolution, a multi-campus research program funded by the University of California Office of Research.

This research has made use of the NASA/IPAC Extragalactic Database (NED) which is operated by the Jet Propulsion Laboratory, California Institute of Technology, under contract with the National Aeronautics and Space Administration. This paper uses data products produced by the OIR Telescope Data Center, supported by the Smithsonian Astrophysical Observatory.

\section{REFERENCES}

Alexander, D. M., \& Hickox, R. C. 2012, NewAR, 56, 93

Alonso-Herrero, A., Engelbracht, C. W., Rieke, M. J., Rieke, G. H., \& Quillen, A. C. 2001, ApJ, 546, 952

Alonso-Herrero, A., García-Marín, M., Rodríguez-Zaurín, J., et al. 2010, A\&A, 522, A7

Alonso-Herrero, A., Pereira-Santaella, M., Rieke, G. H., \& Rigopoulou, D. 2012, ApJ, 744, 2

Alonso-Herrero, A., Rieke, G. H., Rieke, M. J., \& Scoville, N. Z. 2000, ApJ, 532,845

Alonso-Herrero, A., Rieke, G. H., Rieke, M. J., et al. 2006, ApJ, 650, 835

Armus, L., Mazzarella, J. M., Evans, A. S., et al. 2009, PASP, 121, 559

Bedregal, A. G., Colina, L., Alonso-Herrero, A., \& Arribas, S. 2009, ApJ, 698, 1852

Bellocchi, E., Arribas, S., \& Colina, L. 2012, A\&A, 542, 54

Brandl, B., Bernard-Salas, J., Spoon, H. W. W., et al. 2006, ApJ, 653, 1129

Dasyra, K. M., Ho, L. C., Armus, L., et al. 2008, ApJL, 674, L9

Dasyra, K. M., Ho, L. C., Netzer, H., et al. 2011, ApJ, 740, 94
Dasyra, K. M., Tacconi, L. J., Davies, R. I., et al. 2006, ApJ, 651, 835

Davies, R. I., Müller Sánchez, F., Genzel, R., et al. 2007, ApJ, 671, 1388

Diamond-Stanic, A. M., \& Rieke, G. H. 2010, ApJ, 724, 140

Diamond-Stanic, A. M., \& Rieke, G. H. 2012, ApJ, 746, 168

Díaz-Santos, T., Charmandaris, V., Armus, L., et al. 2010, ApJ, 723, 993

Fabricant, D., Cheimets, P., Caldwell, N., \& Geary, J. 1998, PASP, 110, 79

Garcia-Rissmann, A., Vega, L. R., Asari, N. V., et al. 2005, MNRAS, 359 , 765

Gebhardt, K., Kormendy, J., Ho, L. C., et al. 2000, ApJL, 543, L5

González Delgado, R. M., Heckman, T., Leitherer, C., et al. 1998, ApJ, 505,174

Gorjian, V., Cleary, K., Werner, M. W., \& Lawrence, C. R. 2007, ApJL, $655, \mathrm{~L} 73$

Greene, J. E., \& Ho, L. C. 2005, ApJ, 627, 721

Gu, Q., Melnick, J., Cid Fernandes, R., et al. 2006, MNRAS, 366, 480

Gültekin, K., Richstone, D. O., Gebhardt, K., et al. 2009, ApJ, 698, 198

Häring, N., \& Rix, H. W. 2004, ApJL, 604, L89

Hattori, T., Yoshida, M., Ohtani, H., et al. 2004, AJ, 127, 736

Heckman, T. M., Kauffmann, G., Brinchmann, J., et al. 2004, ApJ, 613,109

Hinz, J. L., \& Rieke, G. H. 2006, ApJ, 646, 872

Ho, L., \& Keto, E. 2007, ApJ, 658, 314

Ho, L. C., Green, J. E., Filippenko, A. V., \& Sargent, W. L. W. 2009, ApJS, 183,1

Hopkins, P. F. 2012, MNRAS, 420, L8

Hopkins, P. F., Hernquist, L., Cox, T. J., \& Keres, D. 2008, ApJS, 175,356

Hopkins, P. F., \& Quataert, E. 2010, MNRAS, 407, 1529

Houck, J. R., Roellig, T. L., van Cleve, J., et al. 2004, ApJS, 154, 18

Imanishi, M., Nakagawa, T., Shirahata, M., Ohyama, Y., \& Onaka, T. 2010, ApJ, 721,1233

Jones, D. H., Read, M. A., Saunders, W., et al. 2009, MNRAS, 399, 683

Jones, D. H., Saunders, W., Colless, M., et al. 2004, MNRAS, 355, 747

Kaviraj, S. 2009, MNRAS, 394, 1167

Kennicutt, R. C., Jr. 1998, ARA\&A, 36, 189

Kormendy, J., Bender, R., \& Cornell, M. E. 2011, Natur, 469, 374

Kroupa, P. 2002, Sci, 295, 82

Lynden-Bell, D. 1969, Natur, 223, 690

Magorrian, J., Tremaine, S., Richstone, D., et al. 1998, AJ, 115, 2285

Maiolino, R., \& Rieke, G. H. 1995, ApJ, 454, 95

Marconi, A., \& Hunt, L. K. 2003, ApJL, 589, L21

Marconi, A., Risaliti, G., Gilli, R., et al. 2004, MNRAS, 351, 169

Márquez, I., Moles, M., \& Masegosa, J. 1996, A\&A, 310, 401

Murphy, T. W., Soifer, B. T., Matthews, K., \& Armus, L. 2001, ApJ, 559,201

Nardini, E., Risaliti, G., Watabe, Y., Salvati, M., \& Sani, E. 2010, MNRAS, 405,2505

Onken, C. A., Ferrarese, L., Merritt, D., et al. 2004, ApJ, 615, 645

Pereira-Santaella, M. 2012, PhD thesis, Universidad Autónoma de Madrid

Pereira-Santaella, M., Alonso-Herrero, A., Rieke, G. H., et al. 2010a, ApJS, 188,447

Pereira-Santaella, M., Alonso-Herrero, A., Santos-Lleo, M., et al. 2011, A\&A, 535, A93

Pereira-Santaella, M., Diamond-Stanic, A. M., Alonso-Herrero, A., \& Rieke, G. H. 2010b, ApJ, 725, 2270

Petric, A. O., Armus, L., Howell, J., et al. 2011, ApJ, 730, 28

Poggianti, B. M., \& Wu, H. 2000, ApJ, 529, 157

Rieke, G. H., Alonso-Herrero, A., Werner, B. J., et al. 2009, ApJ, 692, 556

Roche, P. F., Aitken, D. K., Smith, C. H., \& Ward, M. J. 1991, MNRAS, 248, 606

Rodríguez-Zaurín, J., Arribas, S., Monreal-Ibero, A., et al. 2011, A\&A, 527,60

Rodríguez-Zaurín, J., Tadhunter, C., \& González Delgado, R. M. 2010, MNRAS, 403, 1317

Sandage, A., \& Tammann, G. A. 1987, A Revised Shapley-Ames Catalog of Bright Galaxies (2nd ed.; Washington, DC: Carnegie Institution of Washington)

Sanders, D., \& Ishida, C. 2004, in ASP Conf. Ser. 320, The Neutral ISM in Starburst Galaxies, ed. S. Aalto, S. Huttemeister, \& A. Pedlar (San Francisco, CA: ASP), 230

Sanders, D. B., Mazzarella, J. M., Kim, D.-C., Surace, J. A., \& Soifer, B. T. 2003, AJ, 126, 1607

Sanders, D. B., \& Mirabel, F. I. 1996, ARA\&A, 34, 749

Sanders, D. B., Soifer, B. T., Elias, J. H., et al. 1988, ApJ, 325, 74

Schawinski, K., Urry, M. C., Virani, S., et al. 2010, ApJ, 711, 284

Schawinski, K., Virani, S., Simmons, V., et al. 2009, ApJL, 692, L19

Shier, L. M., \& Fisher, J. 1998, ApJ, 497, 163 
Smith, J. D. T., Draine, B. T., Dale, D. A., et al. 2007, ApJ, 656, 770

Spoon, H. W. W., \& Holt, J. 2009, ApJL, 702, L42

Spoon, H. W. W., Marshall, J. A., Houck, J. R., et al. 2007, ApJL, 654, L49

Tacconi, L. J., Genzel, R., Lutz, D., et al. 2002, ApJ, 580, 73

Tokarz, S., \& Roll, J. 1997, in ASP Conf. Ser. 125, Astronomical Data Analysis Software and Systems VI, ed. G. Hunt \& H. E. Payne (San Francisco, CA: ASP), 140
Veilleux, S., Kim, D.-C., Sanders, D. B., Mazzarella, J. M., \& Soifer, B. T. 1995, ApJS, 98, 171

Veilleux, S., Rupke, D. S. N., Kim, D.-C., et al. 2009, ApJS, 182, 628

Wild, V., Heckman, T., \& Charlot, S. 2010, MNRAS, 405, 933

Wilson, A. S., Baldwin, J. A., Sun, S.-D., \& Wright, A. E. 1986, ApJ, 310,121

Yuan, T.-T., Kewley, L. J., \& Sanders, D. B. 2010, ApJ, 709, 884 